University of Nebraska - Lincoln

DigitalCommons@University of Nebraska - Lincoln

\title{
The Sensitivity of Convective Initiation to the Lapse Rate of the Active Cloud-Bearing Layer
}

Adam L. Houston

Dev Niyogi

Follow this and additional works at: https://digitalcommons.unl.edu/geosciencefacpub

Part of the Earth Sciences Commons

This Article is brought to you for free and open access by the Earth and Atmospheric Sciences, Department of at DigitalCommons@University of Nebraska - Lincoln. It has been accepted for inclusion in Papers in the Earth and Atmospheric Sciences by an authorized administrator of DigitalCommons@University of Nebraska - Lincoln. 


\title{
The Sensitivity of Convective Initiation to the Lapse Rate of the Active Cloud-Bearing Layer
}

\author{
ADAM L. HOUSTON \\ Department of Geosciences, University of Nebraska at Lincoln, Lincoln, Nebraska \\ Dev NiYOGi \\ Departments of Agronomy and Earth and Atmospheric Sciences, Purdue University, West Lafayette, Indiana
}

(Manuscript received 25 August 2006, in final form 8 December 2006)

\begin{abstract}
Numerical experiments are conducted using an idealized cloud-resolving model to explore the sensitivity of deep convective initiation (DCI) to the lapse rate of the active cloud-bearing layer [ACBL; the atmospheric layer above the level of free convection (LFC)]. Clouds are initiated using a new technique that involves a preexisting airmass boundary initialized such that the (unrealistic) adjustment of the model state variables to the imposed boundary is disassociated from the simulation of convection. Reference state environments used in the experiment suite have identical mixed layer values of convective inhibition, CAPE, and LFC as well as identical profiles of relative humidity and wind. Of the six simulations conducted for the experiment set, only the three environments with the largest ACBL lapse rates support DCI. The simulated deep convection is initiated from elevated sources (parcels in the convective clouds originate near $1300 \mathrm{~m}$ ) despite the presence of a surface-based boundary. Thermal instability release is found to be more likely in the experiments with larger ACBL lapse rates because the forced ascent at the preexisting boundary is stronger (despite nearly identical boundary depths) and because the parcels' LFCs are lower, irrespective of parcel dilution. In one experiment without deep convection, DCI failure occurs even though thermal instability is released. Results from this experiment along with the results from a heuristic Lagrangian model reveal the existence of two convective regimes dependent on the environmental lapse rate: a supercritical state capable of supporting DCI and a subcritical state that is unlikely to support DCI. Under supercritical conditions the rate of increase in buoyancy due to parcel ascent exceeds the reduction in buoyancy due to dilution. Under subcritical conditions, the rate of increase in buoyancy due to parcel ascent is outpaced by the rate of reduction in buoyancy from dilution. Overall, results demonstrate that the lapse rate of the ACBL is useful in diagnosing and/or predicting DCI.
\end{abstract}

\section{Introduction}

The initiation of deep convection requires (at a minimum) conditional instability (a vertical profile of temperature that can yield the release of thermal instability given parcel saturation) and a trigger (the initial upward motion that releases the thermal instability). A localized trigger for deep convective initiation (DCI) would be unnecessary if the atmosphere was absolutely unstable, but this is usually not the case and more often the atmosphere is characterized by a layer of poten-

Corresponding author address: Dr. Adam L. Houston, Dept. of Geosciences, University of Nebraska at Lincoln, 214 Bessey Hall, Lincoln, NE 68588.

E-mail: ahouston2@unl.edu tially warm air above the surface [quantified as convective inhibition (CIN)] that inhibits the spontaneous release of thermal instability. However, in situations for which atmospheric preconditioning (Johnson and Mapes 2001) has removed CIN, DCI is still not assured since the dilution of individual air parcels ascending toward the level of free convection (LFC) can increase the actual inhibition of each parcel (Ziegler and Rasmussen 1998). This increase in inhibition typically manifests itself as a cooling of cloudy parcels produced when dilution/entrainment reduces parcel moisture, thereby promoting evaporation. Assessing the dilution of individual air parcels requires relaxing parcel theory, which specifically excludes mixing. In doing so, the parcel LFC becomes a time-dependent quantity that increases with increasing parcel dilution (a parcel cooled

DOI: 10.1175/MWR3449.1 

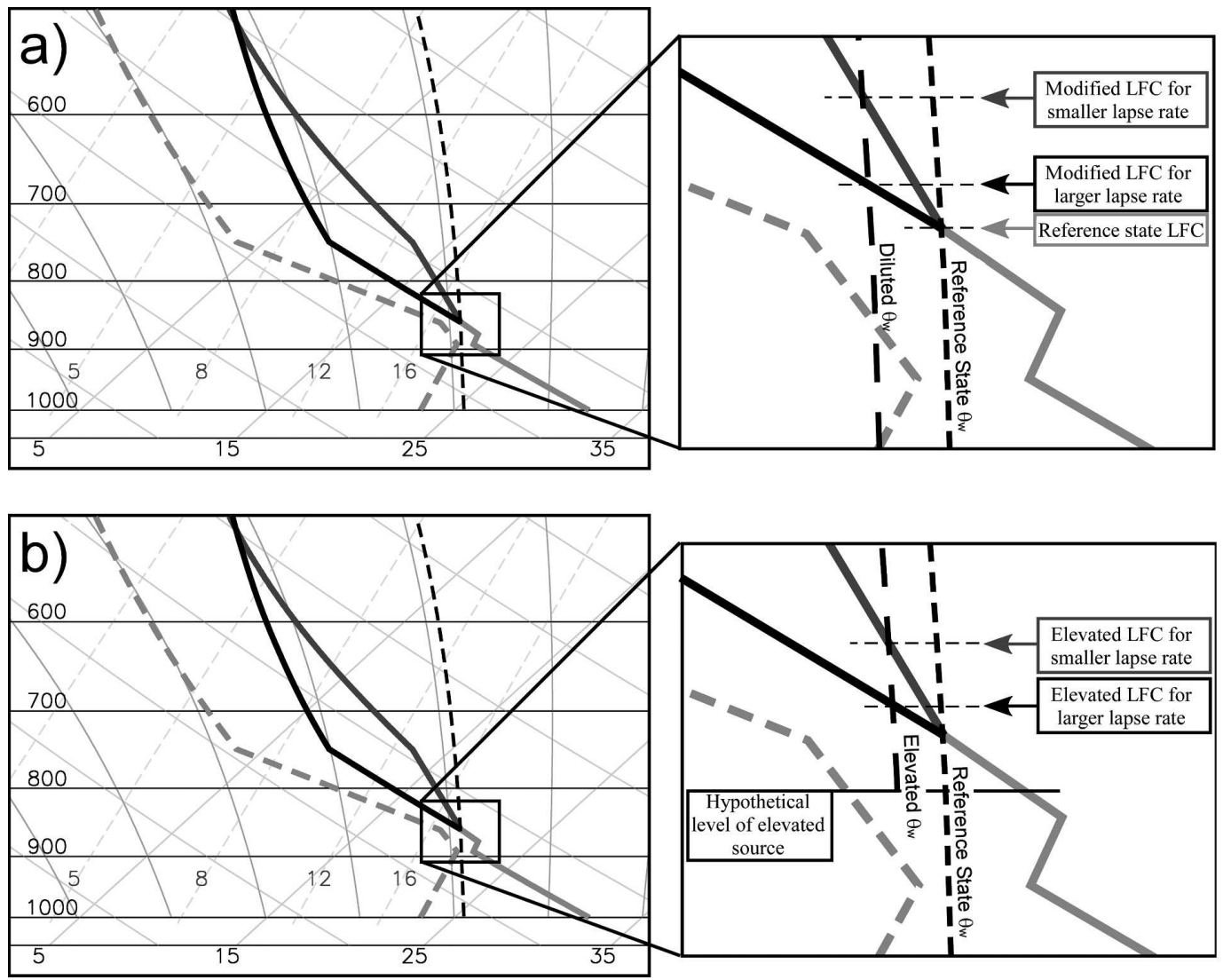

FIG. 1. Illustration of the possible sensitivity of parcel LFC (and by extension DCI) to the lapse rate of the ACBL for (a) parcels originating in a well-mixed boundary layer and (b) parcels originating in an elevated layer.

by dilution must be lifted higher to be warmer than the environment). When parcel theory is relaxed and the evolutions of individual parcels comprising a convective updraft are considered, deep convective initiation is seen to ultimately depend upon the difference between the depth of forced ascent by the trigger and the diluted, time-dependent LFCs of individual parcels: if the diluted (higher) LFC is greater than the depth of the forced ascent, then DCI will not occur.

Since deep convection can also be initiated from elevated sources (parcels originating above the mixed boundary layer), a robust approach for assessing the likelihood of DCI must also consider this source. Even if dilution is neglected and parcel theory is valid, the use of surface-based or even mixed-layer-based values of CIN and LFC would neglect the possibility that the depth of the forced ascent of elevated parcels could exceed their LFCs. By considering the difference between the depth of the forced ascent by the trigger and the LFCs of individual parcels (diluted or not), it is unnecessary to assume a parcel source ex ante facto.

It is only by casting the regulation of DCI in terms of individual parcels that the possible sensitivity of DCI to the environmental lapse rate of the active cloudbearing layer can be considered. [The environmental lapse rate is defined as $\Gamma=-\partial T_{0} / \partial z$, where $T_{0}$ is the environmental temperature, and the ACBL is defined as the layer above the LFC, that is, where "active" (Stull 1985) deep convection occurs.] For parcels originating in the planetary boundary layer the increase in the LFC through dilution should be larger for smaller ACBL lapse rates. Consider, for example, two environmental temperature profiles that are identical below a common mixed-layer-based LFC (ML-LFC) but have different lapse rates above this level (Fig. 1a). Dilution of a parcel ascending out of a well-mixed boundary layer will cause the wet-bulb potential temperature $\left(\theta_{w}\right)$, which is generally conserved in the absence of mixing, to decrease. This decrease can be driven by the evaporative cooling of cloudy air associated with the mixing of less moist air into the parcel (Ziegler et al. 1997; Ziegler and Rasmussen 1998). This dilution will increase the LFC but the amount of increase will depend on the environmental lapse rate of the ACBL 
(Fig. 1a): for a "small" ACBL lapse rate, the environmental temperature above the ML-LFC will be warmer than that for a "large" ACBL lapse rate; thus, a diluted parcel must be lifted higher before it can become positively buoyant. In other words, the diluted LFC will be higher for a smaller lapse rate than for a large lapse rate and DCI less likely.

As with parcels originating in a well-mixed boundary layer, parcels originating in elevated layers should also be characterized by LFCs that are higher for smaller ACBL lapse rates. For two environmental temperature profiles with identical ML-LFCs (Fig. 1b), the moist adiabat (line of constant wet-bulb potential temperature) of an elevated source will intersect the environmental temperature at a higher elevation for a smaller ACBL lapse rate. Thus, the parcel LFC will be higher for a smaller lapse rate. This characteristic would not require parcel dilution.

In an effort to understand the possible sensitivity of DCI to the environmental lapse rate of the ACBL, idealized, cloud-scale, numerical experiments have been conducted in which the response of cloud growth to various environmental lapse rates was tested. Variations in the lapse rates of the reference state (1D) vertical profiles used in the experiments were imposed using an analytical function that ensures constant values of mixed-layer-based CIN (ML-CIN), CAPE (MLCAPE), LFC, and relative humidity between the different reference states.

Cloud initiation has been simulated in each experiment using a prescribed airmass boundary. While DCI has been attributed to phenomena other than airmass boundaries, the ubiquity of boundary-initiated deep convection (Purdom 1982; Wilson and Schreiber 1986; Wilson and Roberts 2006) suggests that this experimental design will maximize the relevance of the findings. Nevertheless, it is most accurate to interpret the results presented herein as representing the sensitivity of deep convection initiated on preexisting boundaries to the environmental lapse rate in the cloud-bearing layer.

The realistic initiation of deep convection in an idealized framework has been particularly challenging in the study of DCI (Ziegler et al. 1997). A method of convective initiation has been developed for this work that we believe satisfies the dual constraints of controllability (necessary for systematically conducting experiments) and realism. An explanation of this method along with a brief description of the numerical model, definition of the experimental suite of analytical reference state environments, and discussion of the limitations of simulated 2D deep convection will follow in section 2. Experimental results and discussion are presented in section 3 followed by conclusions in section 4 .

\section{Experimental design}

\section{a. Model description}

All numerical experiments conducted for this work have been performed with the Illinois Collaborative Model for Multiscale Atmospheric Simulations (ICOMMAS). ICOMMAS is a successor to COMMAS (Wicker and Wilhelmson 1995), which has been used extensively in the past for detailed examinations of cloud-scale phenomena (Wicker and Wilhelmson 1995; Wicker et al. 1997; Gilmore and Wicker 1998; Atkins et al. 1999; Peckham and Wicker 2000). The kernel of ICOMMAS is similar in many respects to COMMAS though three primary differences distinguish the two models: (i) the temporal discretization of lowfrequency tendencies involves the third-order RungeKutta (RK3) method instead of a forward-in-time (Wicker and Wilhelmson 1995) or second-order Runge-Kutta (RK2) (Wicker and Skamarock 1998) scheme; (ii) high-order ( $\geq$ third order) approximations are used for the spatial discretization of advection instead of the second-order approximation used in the original version of COMMAS; and (iii) the Straka three-phase ice parameterization (Gilmore et al. 2004a) is utilized in lieu of the Kessler or Tao schemes.

ICOMMAS was developed in parallel with L. J. Wicker's development of the National Severe Storms Laboratory version of COMMAS (NCOMMAS; Coniglio et al. 2006) and shares many features. A full description of ICOMMAS is documented by Houston (2004)

As with COMMAS and NCOMMAS, ICOMMAS neglects orography, solar radiation, and surface fluxes of heat and moisture. Furthermore, as is often the case in idealized modeling studies, surface friction is neglected (the lower boundary is free slip). The model domain for all experiments is $40 \mathrm{~km}$ long and $20 \mathrm{~km}$ deep with a horizontal gridpoint spacing of $100 \mathrm{~m}$ and a vertical gridpoint spacing of $50 \mathrm{~m}$ stretched to $125 \mathrm{~m}$. The grid spacing used for this work is far too coarse to explicitly treat turbulent kinetic energy dissipation; thus, a version of the 1.5-order closure turbulence parameterization of Klemp and Wilhelmson (1978) is used to approximate the subgrid-scale kinetic energy dissipation. The Klemp and Wilhelmson implementation utilizes a formulation of the prognostic equation for turbulent kinetic energy (TKE) along with fluxgradient theory to predict an eddy-mixing coefficient. Mixing-length theory is then used with this mixing coefficient to close the subgrid-scale transports.

The tunable model parameter values used for these experiments are largely the same as the values de- 


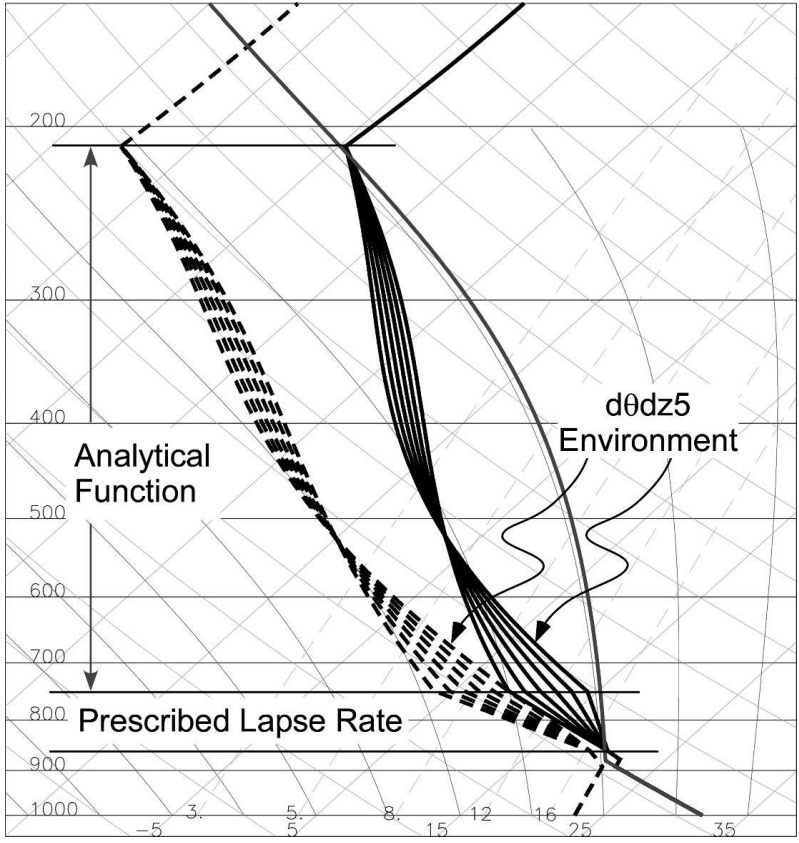

FIG. 2. Skew $T-\log p$ diagram of temperature (continuous black curves) and water vapor mixing ratio (broken black curves) for each of the reference state environments used in the experiment suite.

scribed by Houston (2004); however, there are two primary differences. The first difference is that the current model setup is $2 \mathrm{D}$. The potential limitations of the $2 \mathrm{D}$ framework are reviewed in section $2 \mathrm{~d}$. The second difference is in the microphysical parameterization. In the current experiment set, the hail/graupel density and slope intercept are $400 \mathrm{~kg} \mathrm{~m}^{-3}$ and $4 \times 10^{8}$, respectively. These values correspond to the "small graupel" settings used by Gilmore et al. (2004b). A microphysical treatment weighted toward small graupel was chosen because the numerical experiments herein are designed to simulate primarily the early stages of deep convection when significant quantities of large hail are limited.

\section{b. Reference state}

A systematic examination of the sensitivity of DCI to lapse rate requires a carefully designed parameter space that isolates the effect of changes in lapse rate from other environmental characteristics that can modulate DCI. To this end, a technique was designed to analytically generate vertical profiles of temperature, moisture, and winds.

The reference state in ICOMMAS is horizontally homogeneous and is imposed by introducing a reference state "sounding" containing the vertical distributions of the temperature, moisture, and winds. Six soundings are used and are illustrated in Fig. 2. The temperature
TABLE 1. Thermodynamic characteristics within the prescribed lapse rate layers for each of the six experiments conducted for this work.

\begin{tabular}{lcc}
\hline \hline Expt & $d \theta / d z\left(\mathrm{~K} \mathrm{~km}^{-1}\right)$ & $\Gamma=-d T / d z\left(\mathrm{~K} \mathrm{~km}^{-1}\right)$ \\
\hline $\mathrm{d} \theta \mathrm{dz} 0$ & 0 & 9.8 \\
$\mathrm{~d} \theta \mathrm{dz} 1$ & 1 & 8.8 \\
$\mathrm{~d} \theta \mathrm{dz2}$ & 2 & 7.9 \\
$\mathrm{~d} \theta \mathrm{dz3}$ & 3 & 6.9 \\
$\mathrm{~d} \theta \mathrm{dz} 4$ & 4 & 6.0 \\
$\mathrm{~d} \theta \mathrm{dz5}$ & 5 & 5.1 \\
\hline
\end{tabular}

and moisture profiles of these soundings are identical below the $M L-L F C$ at $1320 \mathrm{~m}$ and include an inversion between $1000 \mathrm{~m}$ and the ML-LFC that yields approximately $5.1 \mathrm{~J} \mathrm{~kg}^{-1}$ of ML-CIN.

Lapse rate variations between the soundings of the experiment set are imposed through modification of the temperature profile above the common ML-LFC. In each sounding, the prescribed lapse rate occupies the layer between the ML-LFC and $2500 \mathrm{~m}$. Values of $d \theta_{0} / d z$ for these soundings range from 0 to $5 \mathrm{~K} \mathrm{~km}^{-1}$ (which correspond to lapse rates ranging from 9.8 to 5.1 $\mathrm{K} \mathrm{km}^{-1}$ ). A summary of the six experiments that compose the experiment set appears in Table 1 . Note that the experiment name refers to the value of $d \theta_{0} / d z$ within the prescribed lapse rate (e.g., d $\theta \mathrm{dz} 0$ corresponds to a value of $d \theta_{0} / d z$ equal to 0 within the prescribed lapse rate layer).

To ensure identical values of ML-CAPE and tropopause height across all vertical profiles, the temperature profile above the prescribed lapse rate layer is defined by prescribing the vertical distribution of buoyancy using an analytical function that relates the MLCAPE within the prescribed lapse rate layer (which will vary for each sounding) to the predefined total MLCAPE of the sounding. The total ML-CAPE for all soundings is fixed at a value of $2500 \mathrm{~J} \mathrm{~kg}^{-1}$. A full description of the approach is included in appendix A.

The profiles of moisture for each environment are determined after the prescription of temperature and are set to insure that the relative humidity above the ML-LFC is the same for each environment. ${ }^{1}$ As indicated in Fig. 2, the $\theta_{w}$ value within the cloud-bearing layer below $\sim 5300 \mathrm{~m}$ is anticorrelated with the lapse rate of the ACBL. This source of low- $\theta_{w}$ air could increase the magnitude of cloud dilution and thereby

\footnotetext{
${ }^{1}$ For simplicity, the analytical buoyancy is computed without accounting for the effects of moisture on density. Such effects are most significant in the low levels of the atmosphere where the amount of moisture is largest. Since the reference state thermodynamics below the ML-LFC are identical between soundings used in this work, neglecting moisture in the buoyancy has little effect on the CAPE.
} 


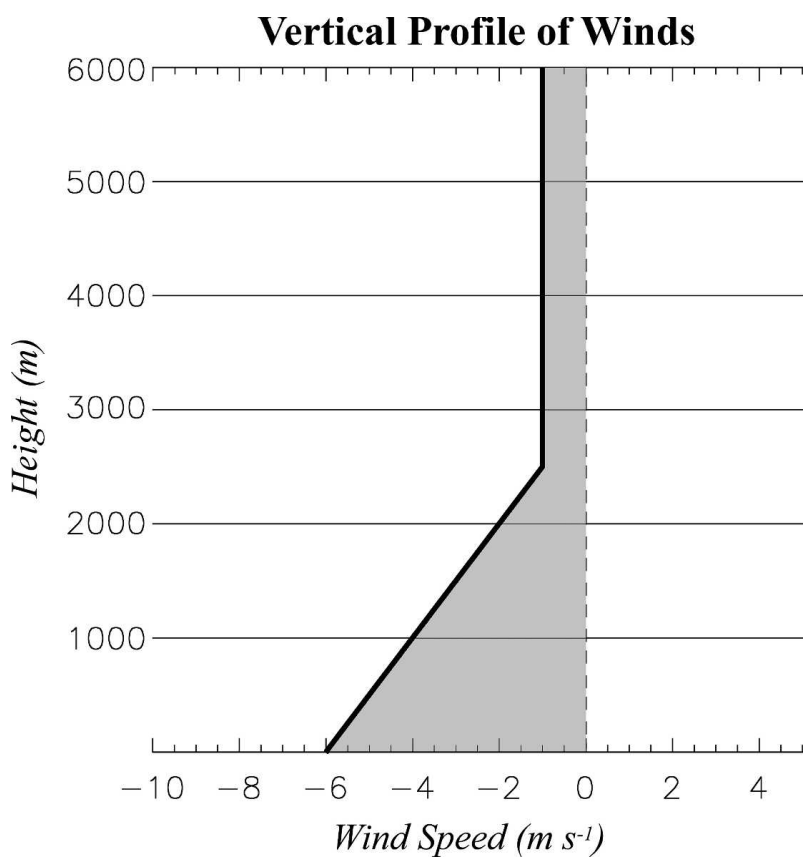

FIG. 3. Vertical profile of reference state winds used for all experiments.

mitigate the hypothesized increased likelihood of DCI in the higher lapse rate environments.

The vertical profile of wind velocity is the same for all soundings and is illustrated in Fig. 3. Positive vertical shear $(d u / d x>0)$ was included in the reference state winds because of the well-documented correlation between the depth-magnitude of forced ascent at airmass boundaries and the vertical shear (e.g., Rotunno et al. 1988; Xu 1992; Liu and Moncrieff 1996; Xu et al. 1996; Xue et al. 1997; Weisman and Rotunno 2004). The value for the vertical wind shear was chosen so that the environment would not possess a steering level (a level at which the airmass boundary-relative winds are zero). This ensured that clouds developing at the boundary would be transported rearward of the boundary. The imposed vertical wind shear vector was directed toward the east and had a magnitude of $2 \times 10^{-3} \mathrm{~s}^{-1}$. This wind shear magnitude is well below that which would be expected for supercells. Because of the inherent threedimensionality of supercells, a more robust comparison can be made between the $2 \mathrm{D}$ deep moist convection modeled in this work to real deep moist convection in environments that are not supercellular. A summary of the parameters shared by all six experiments of the control experiment set are listed in Table 2.

\section{c. Preexisting airmass boundary initialization}

Deep convection is traditionally initiated in idealized cloud models with a thermal bubble: a localized region
TABLE 2. Parameter values common to each environment used in these experiments.

\begin{tabular}{lcc}
\hline \hline \multicolumn{1}{c}{ Parameter } & & Value \\
\hline $\begin{array}{l}\text { Total CAPE of final sounding } \\
\text { "Boundary layer" potential temperature }\end{array}$ & $E_{\text {tot }}$ & $\begin{array}{c}2500 \mathrm{~J} \mathrm{~kg}^{-1} \\
305 \mathrm{~K}\end{array}$ \\
CIN & & $5.1 \mathrm{~J} \mathrm{~kg}^{-1}$ \\
$\begin{array}{l}\text { Height of the tropopause (top of the } \\
\text { analytical layer) }\end{array}$ & $z_{t}$ & $12000 \mathrm{~m}$ \\
$\begin{array}{l}\text { Top of the prescribed lapse rate layer } \\
\text { Top of the layer with vertical wind shear }\end{array}$ & $z_{m}$ & $2500 \mathrm{~m}$ \\
$\begin{array}{l}\text { Relative humidity at the top of the } \\
\text { prescribed lapse rate layer }\end{array}$ & $2500 \mathrm{~m}$ \\
$\begin{array}{l}\text { Relative humidity at the tropopause } \\
\text { Magnitude of the nonzero vertical wind } \\
\text { shear }\end{array}$ & & $70 \%$ \\
\end{tabular}

in which the temperature and/or moisture are perturbed so as to locally release thermal instability. While inherently unrealistic, this method is usually satisfactory when the perturbation is small and when the focus of the analysis is on the character of deep convection several convective time scales following initiation. However, when the focus of the analysis is on initiation, the thermal bubble is inappropriate (Ziegler et al. 1997).

Alternatives to the thermal bubble in idealized cloud-scale models have generally relied upon airmass boundaries to initiate deep convection. In these approaches, the mechanical lifting and/or a solenoidal circulation along the imposed boundary trigger deep convection. Previous methods can be loosely categorized as either ad hoc initializations in which the temperature, moisture, and/or wind field are explicitly imposed (e.g., Hane 1975; Lee and Wilhelmson 1997; Atkins et al. 1999; Peckham et al. 2004) or forcing initializations in which temperature, moisture, and/or wind velocity heterogeneities are created gradually via a tendency term (e.g., Dudhia and Moncrieff 1989). Both categories of initialization can be criticized for lack of realism but, as with the thermal bubble initialization, such deficiencies can largely be ignored when the focus of the analysis is on the character of the simulated deep convection well after initiation.

A preexisting boundary has been chosen for the initiation of deep convection in these experiments. DCI is frequently attributed to airmass boundaries (Purdom 1982; Wilson and Schreiber 1986; Wilson and Roberts 2006) and thus this mechanism has broad applicability to the study of deep convective initiation.

The preexisting airmass boundary used for these experiments is initialized as a density current. The vertical structure of a density current (e.g., the depth, magnitude of ascent, etc.) is dependent on the vertical wind shear (Xu 1992; Liu and Moncrieff 1996; Xu et al. 1996; Xue et al. 1997) and static stability (Liu and Moncrieff 


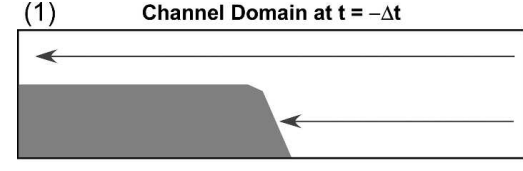

(3)

Full Domain at $\mathrm{t}=\mathbf{0}$

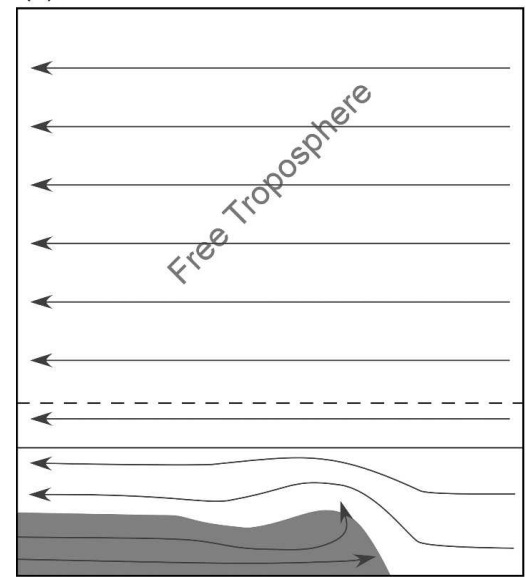

FIG. 4. Conceptual illustration of the airmass boundary initialization ("seeding") technique utilized in this work. Each panel represents a step in the procedure.

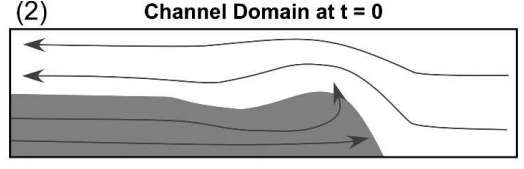

(4) Full Domain at $\mathbf{t}=+\Delta \mathrm{t}$

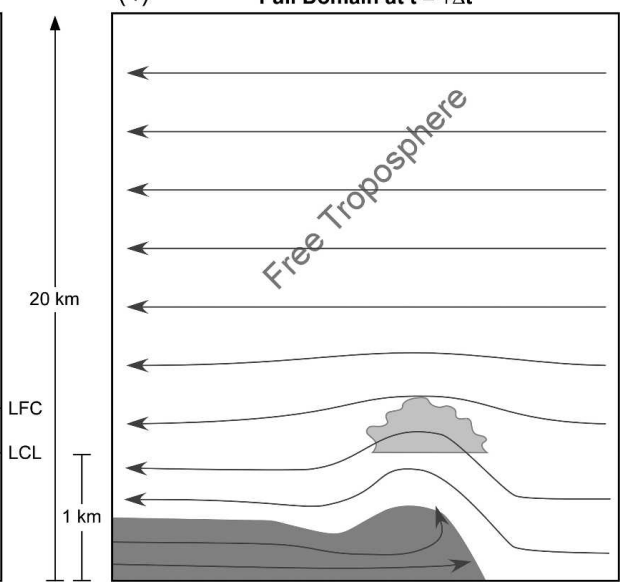

2000; Xue 2002) of the reference state environment upstream of the density current. In these experiments the vertical shear and static stability of the well-mixed layer within which the shallow density current resides are the same for all environments; thus, the vertical structures of the simulated preexisting boundaries should be as well.

The preexisting boundary is initialized as a perturbation to the 1D (stratified) reference state potential temperature and wind velocity. The initial block is $550 \mathrm{~m}$ deep and covers the western $10 \mathrm{~km}$ of the domain. The air mass within the block is initially static and $4 \mathrm{~K}$ colder than the reference state conditions.

The model's state variables will vigorously adjust to the imposed boundary. This initial adjustment to the block is unrealistic: a block of cold air does materialize suddenly like this in the actual atmosphere. This adjustment has the potential to produce an unrealistic treatment of convective initiation. To obviate this threat to the realism of the initialization method, this adjustment should occur prior to, and independent from, convective initiation along the boundary. To meet this requirement, a technique has been developed that simulates the adjustment process separately from the simulation of convective initiation.

The key element of this technique is to simulate the preexisting boundary in a shallow (channel) domain prior to introducing the preexisting boundary into the full domain. (The shallow domain can be thought of as a strongly capped boundary layer.) Since the domain captures the portion of the reference environment be- low the LCL, cloud formation is not allowed and because of the reflective upper boundary condition, the flow is purely lateral at the upper boundary. Thus, when the preexisting boundary and its attendant flow field are introduced into the larger domain, the vertical velocity fields at and above the LCL and LFC are able to gradually respond to the preexisting boundary. In other words, the vigorous adjustment of the model's state variables is disassociated from the simulation of convection.

The specific steps in the technique are illustrated in Fig. 4 and are as follows. (i) The block of cold air is initialized in the channel domain. (ii) The model state variables are allowed to adjust within the channel domain for $3600 \mathrm{~s}$. (iii) The temperature and momentum fields associated with the "adjusted" preexisting boundary are used to "seed" the full domain simulation. As illustrated in Fig. 4 and in the results presented in section 3 , the time that the seed is introduced into the full domain is treated as the initial time $(t=0)$. (iv) Additional (gradual) adjustment to the preexisting boundary produces a forced cumulus cloud and the potential for active convection. Because the lowest $1000 \mathrm{~m}$ of all reference state environments used in the control experiment suite are identical, the same seed is used for each experiment.

Adjustment of the model's state variables will occur in response to any changes to the density or wind fields within the air masses on either side of a boundary. However, the vigorous adjustment that this technique aims to manage is an artifact of initializing the bound- 

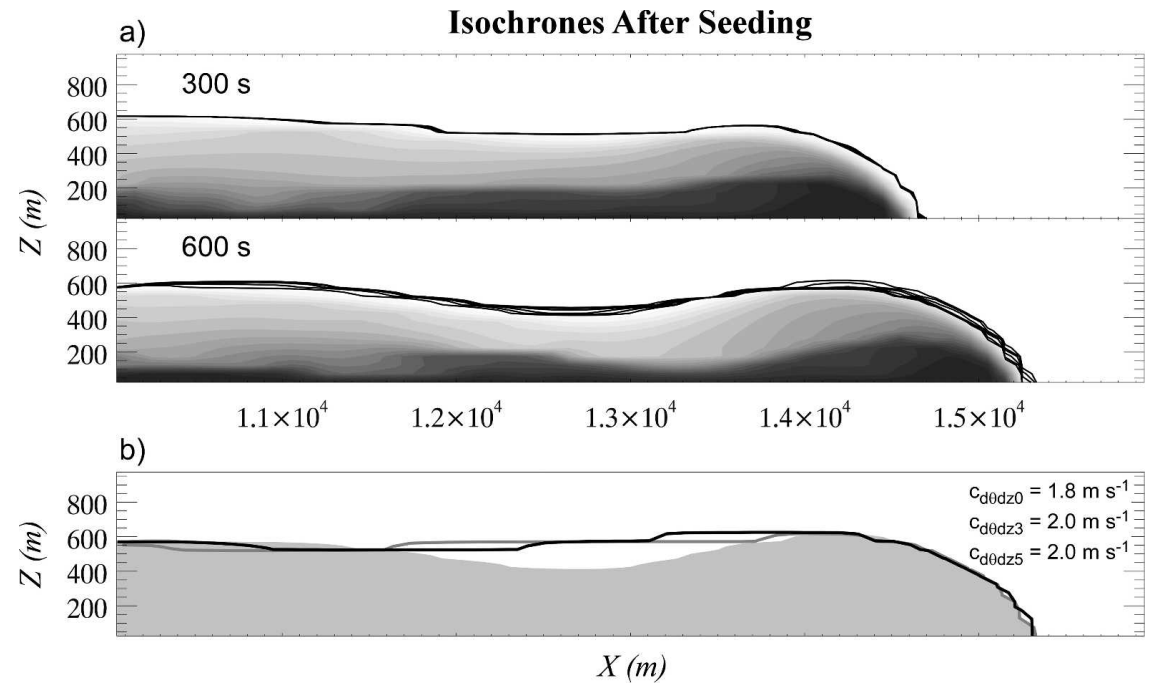

FIG. 5. (a) Isochrones (defined as the $0-\mathrm{K} \theta^{\prime}$ contour) for the preexisting boundaries simulated in each of the six experiments at 300 and $600 \mathrm{~s}$ after seeding (3900 and $4200 \mathrm{~s}$, respectively). The $\theta^{\prime}$ field for the $\mathrm{d} \theta \mathrm{dz} 0$ experiment is shaded at an interval of $0.2 \mathrm{~K}$. (b) Isochrones for the $\mathrm{d} \theta \mathrm{dz} 3$ experiment at $2400 \mathrm{~s}$ (black curve) and the d $\theta \mathrm{dz} 5$ experiment at $3600 \mathrm{~s}$ (gray curve) along with the region of $\theta^{\prime}<0 \mathrm{~K}$ for the $\mathrm{d} \theta \mathrm{dz} 0$ experiment (shaded). The boundary translation speeds at 600,2400 , and $3600 \mathrm{~s}$ for the $\mathrm{d} \theta \mathrm{dz} 0, \mathrm{~d} \theta \mathrm{dz} 3$, and $\mathrm{d} \theta \mathrm{dz} 5$ experiments, respectively, appear in the top-right corner of the panel.

ary with a block of cold air. There are other methods for initializing the air mass on the cold side of the boundary that would avoid this initial unrealistic adjustment. One such method is the heat sink (e.g., Dudhia and Moncrieff 1989; Garner and Thorpe 1992; Fovell and Tan 2000). In this method the air mass on the cool side of the boundary is slowly introduced by cooling a region of the atmosphere in contact with the surface. This approach can be thought of as simulating the evaporative cooling associated with precipitation or the reduction in insolation due to cloud cover. However, while bearing similarities to atmospheric processes, the heat sink method makes no attempt to achieve a quasi-steady-state boundary prior to DCI. Thus, if, as in the simulations conducted for this work, DCI occurs at different times in each experiment, there is no way to ensure that the structure of the boundary is being controlled for. However, in the method used here, not only is the initial unrealistic adjustment of the block isolated from DCI, but by allowing the boundary to evolve for $1 \mathrm{~h}$ before introducing the seed into the full domain, this method also ensures a quasi-steady-state boundary and thus controls for variations in boundary structure.

The steadiness of the simulated boundaries is illustrated with $X-Z$ isochrones in Fig. 5. It is clear that 1) the classic density current structure is captured by the initialization procedure and 2) the structural differences between the density currents are very small. The latter (qualitative) observation is further supported (quantitatively) by noting that the difference between the density current depths of the largest and smallest lapse experiments is $\sim 25 \mathrm{~m}$ after $600 \mathrm{~s}$ of integration. This difference is only $4 \%$ of the density current depth. Furthermore, as it will be shown in section 3, thermal instability release is delayed for more than $2400 \mathrm{~s}$ following the introduction of the seed in the $\mathrm{d} \theta \mathrm{dz} 3$ experiment. Yet, as illustrated in Fig. 5b, the structure and translational speed of the density current remains virtually unchanged throughout the simulation. In the $\mathrm{d} \theta \mathrm{dz} 5$ experiment, for which thermal instability is never released, the density current structure and translational speed remain quasi-steady through the entire simulation.

While this initialization mechanism clearly captures the essence of airmass boundaries that behave as density currents, the omission of heterogeneous surface fluxes of heat and moisture will neglect the planetary boundary layer secondary circulations that can play a role in DCI (refer to the review of Weckwerth and Parsons 2006). However, such boundary layer processes are not necessary for DCI.

\section{d. $2 D$ versus $3 D$ simulations of deep convection}

Real deep convection is rarely slab-symmetric (twodimensional) so there will undoubtedly be some loss of generality using a 2D model framework. Comparisons between 2D (rectilinear) and 3D (either axisymmetric or fully $3 \mathrm{D}$ ) convection have consistently demonstrated 


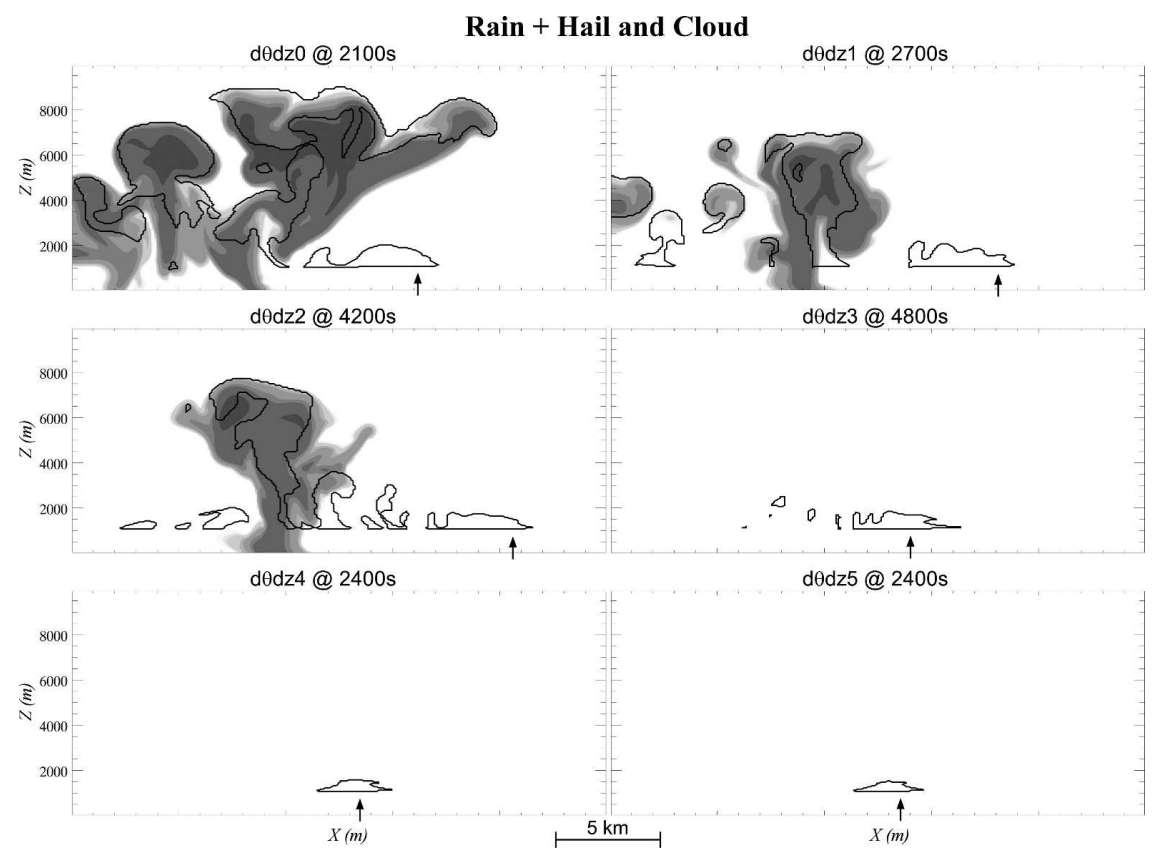

FIG. 6. Precipitation and cloud fields for the six experiments. Precipitation (the combined mixing ratios of rainwater and hail/graupel) is shaded at a logarithmic interval of $5 \times 10^{-n} \mathrm{~kg}$ $\mathrm{kg}^{-1}$ from a minimum value of $5 \times 10^{-7} \mathrm{~kg} \mathrm{~kg}^{-1}$, and the $5 \times 10^{-8} \mathrm{~kg} \mathrm{~kg}^{-1}$ mixing ratio isopleths of the total cloud (the combined mixing ratios of cloud water and cloud ice) are contoured. Vertical black arrows indicate the position of the preexisting boundary. All times are the elapsed times since the introduction of the seed into the full domain.

several limitations of 2D simulations that might be relevant to the experiments conducted for this work.

Midlevel downdrafts on the flanks of a growing cloud and driven by the cloud's buoyant circulation are considerably stronger in 2D clouds (Ogura 1963; Murray 1970; Schlesinger 1984). This disparity is a direct consequence of the unrealistically small area that the downdraft is confined to occupy in 2D. As argued by Schlesinger (1984), if the updraft occupies a given fraction $\sigma$ of the total cell width, then the ratio of downdraft-to-updraft areas in $2 \mathrm{D}$ is a factor of $\sigma /(1+\sigma)$ smaller than the downdraft-to-updraft area ratio in axisymmetric coordinates. The compressional warming and drying associated with this "compensating" downdraft in place just outside of the updraft has the potential to decrease the thermal buoyancy within the cloud and exaggerate the erosion of the cloud column through entrainment (Schlesinger 1984). This cloud column erosion can yield a 2D cloud shaped like a mushroom in situations where no such structure is apparent in 3D (Murray 1970). Given that convective initiation is intimately tied to processes occurring in the early stages of cloud growth, this characteristic of 2D simulations must be considered when interpreting the results from the experiments performed for this work.

Despite these limitations of the 2D framework, 2D simulations of deep convection have been used extensively over the last 10 yr (e.g., Fovell and Dailey 1995; Fovell and Tan 1998; Lin et al. 1998; Bryan and Fritch 2002; Xue 2002; Bryan 2005). In particular, the work of Fovell and coauthors and Lin and coauthors has treated the development of deep convection on airmass boundaries. And while their airmass boundaries were storm generated (not preexisting), their results clearly demonstrated that the 2D framework could capture the initiation of deep convection.

\section{Results and discussion}

Of the six simulations conducted for the experiment set, only the three environments with the largest lapse rates support DCI. ${ }^{2}$ Snapshots of the cloud and precipitation for these six experiments are provided in Fig. 6 (all times listed in this figure and elsewhere in this article are the elapsed times since the introduction of the seed into the full domain). Shallow clouds are simulated at the preexisting boundary in the environments with the three smallest lapse rates but DCI is inhibited through the duration of these simulations.

\footnotetext{
${ }^{2} \mathrm{DCI}$ is defined for this work as moist convection that produces precipitation at the surface.
} 


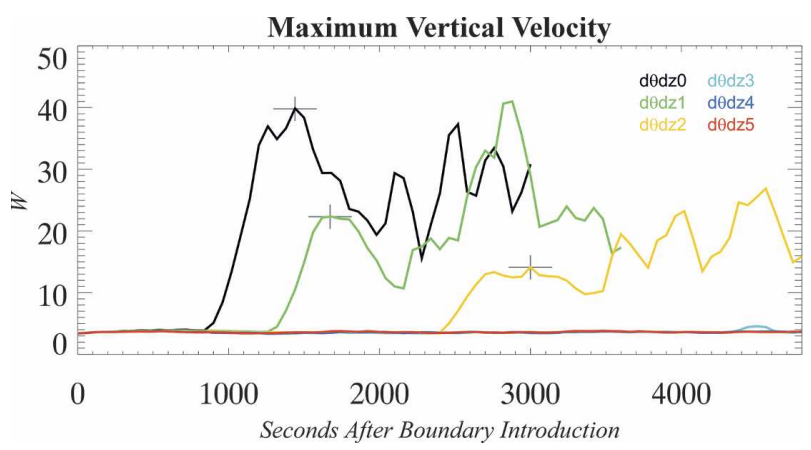

FIG. 7. Time series of domain-maximum vertical velocity values for the six experiments. Crosshairs on the $\mathrm{d} \theta \mathrm{dz} 0, \mathrm{~d} \theta \mathrm{dz} 1$, and $\mathrm{d} \theta \mathrm{dz} 2$ experiments indicate the maximum vertical velocity for the first deep convective cloud of the experiment.

Time series plots of the domain-maximum vertical velocity values (Fig. 7) show that, among the environments that supported DCI, the time required for initiation decreases with increasing lapse rate. Furthermore, the magnitude of the maximum vertical velocity value for the initial deep convection clearly increases with increasing lapse rate.

In general, there are two possible explanations for the simulated sensitivity of DCI to lapse rate: 1) air parcels are less likely to reach their LFC for smaller lapse rate environments or 2) air parcels reach their LFC (thermal instability is released) but the resultant shallow convection becomes diluted prior to maturation for smaller lapse rate environments. Section 3 a will treat the former possibility and section $3 b$ will treat the latter.

\section{a. Dependence of parcel ascent to the LFC on lapse rate}

In these experiments, if air parcels are more likely to reach their LFC in the larger lapse rate environments, it is because the forced ascent at the preexisting boundary is stronger and/or the parcels' LFCs are lower (as proposed in section 1). The following subsections address these two possibilities.

\section{1) INTEREXPERIMENT VARIATIONS IN FORCED ASCENT}

The assumption underpinning the hypothesized role of LFC height in regulating DCI between environments with differing ACBL lapse rates (section 1) was that the depth of the forced ascent was independent of the lapse rate. However, in 2D idealized density current experiments, Xue (2002) found that the depth of the forced ascent at the leading edge of a density current (as well as the depth of the density current itself) was negatively
TABLE 3. Values of wet-bulb potential temperature (K) surfaces chosen for the seven heights for each of the six experiments.

\begin{tabular}{ccccccc}
\hline \hline $\begin{array}{c}\text { Surface } \\
\text { height }(\mathrm{m})\end{array}$ & $\mathrm{d} \theta \mathrm{dz} 0$ & $\mathrm{~d} \theta \mathrm{dz} 1$ & $\mathrm{~d} \theta \mathrm{dz} 2$ & $\mathrm{~d} \theta \mathrm{dz} 3$ & $\mathrm{~d} \theta \mathrm{dz} 4$ & $\mathrm{~d} \theta \mathrm{dz} 5$ \\
\hline 1000 & 298.6 & 298.6 & 298.6 & 298.6 & 298.6 & 298.6 \\
1100 & 298.5 & 298.5 & 298.5 & 298.5 & 298.5 & 298.5 \\
1200 & 298.3 & 298.3 & 298.3 & 298.3 & 298.3 & 298.3 \\
1300 & 298.0 & 298.0 & 298.0 & 298.0 & 298.0 & 298.0 \\
1400 & 297.4 & 297.4 & 297.5 & 297.6 & 297.6 & 297.7 \\
1500 & 296.7 & 296.8 & 297.0 & 297.1 & 297.3 & 297.4 \\
1600 & 296.0 & 296.2 & 296.5 & 296.7 & 296.9 & 297.2 \\
\hline
\end{tabular}

correlated with the lapse rate in an overlying inversion. Thus, it is possible that identical initial preexisting boundaries can produce differing depths of forced ascent if only the overlying lapse rates are different.

To examine the differences in forced ascent in these experiments, we consider the upward displacement of wet-bulb isentropic $\left(\theta_{w}\right)$ surfaces. This method was chosen in place of trajectory analysis because the deflection of $\theta_{w}$ surfaces captures the cumulative effect of parcel displacement. Since the vertical profiles of $\theta_{w}$ differ above the LFC between experiments, the same surfaces could not be used for all experiments and all heights. Instead, a suite of heights was chosen and the $\theta_{w}$ surfaces in each environment that corresponded to these altitudes were identified (see Table 3 for a summary of the $\theta_{w}$ values used for each of the seven heights). Analysis of the vertical displacement of these surfaces was undertaken prior to the release of thermal instability in any of the experiments. After this time, surface displacement could have been the result of positively buoyant motions as well.

The displacement of the chosen surfaces is illustrated in Fig. 8. The black (gray) curves represent the surfaces of the $\mathrm{d} \theta \mathrm{dz} 0(\mathrm{~d} \theta \mathrm{dz} 5)$ experiment. The corresponding surfaces for the remaining experiments reside within the shaded envelopes between the $\mathrm{d} \theta \mathrm{dz} 0$ and $\mathrm{d} \theta \mathrm{dz} 5$ curves and are distributed monotonically within these envelopes. It is clear that the forced ascent at the preexisting boundary increases with increasing ACBL lapse rate. Figure 9 provides a more quantitative assessment of the displacements of the $\theta_{w}$ surfaces. From this figure it is also clear that while the magnitude of the displacement is largest for the lower isentropic surfaces (e.g., the $1000-\mathrm{m}$ isentropic surface in the $\mathrm{d} \theta \mathrm{dz} 0$ experiment is displaced $165 \mathrm{~m}$ but the $1600-\mathrm{m}$ surface is displaced only $104 \mathrm{~m}$ ), the interexperiment differences between the surface displacements are largest for the upper isentropic surfaces (e.g., the difference in the displacement of the $1000-\mathrm{m}$ surface between the $\mathrm{d} \theta \mathrm{dz} 0$ and $\mathrm{d} \theta \mathrm{dz} 1$ experiments is $10 \mathrm{~m}$ but is $10.6 \mathrm{~m}$ for the 1600-m surface). Consistent with this finding, the larg- 


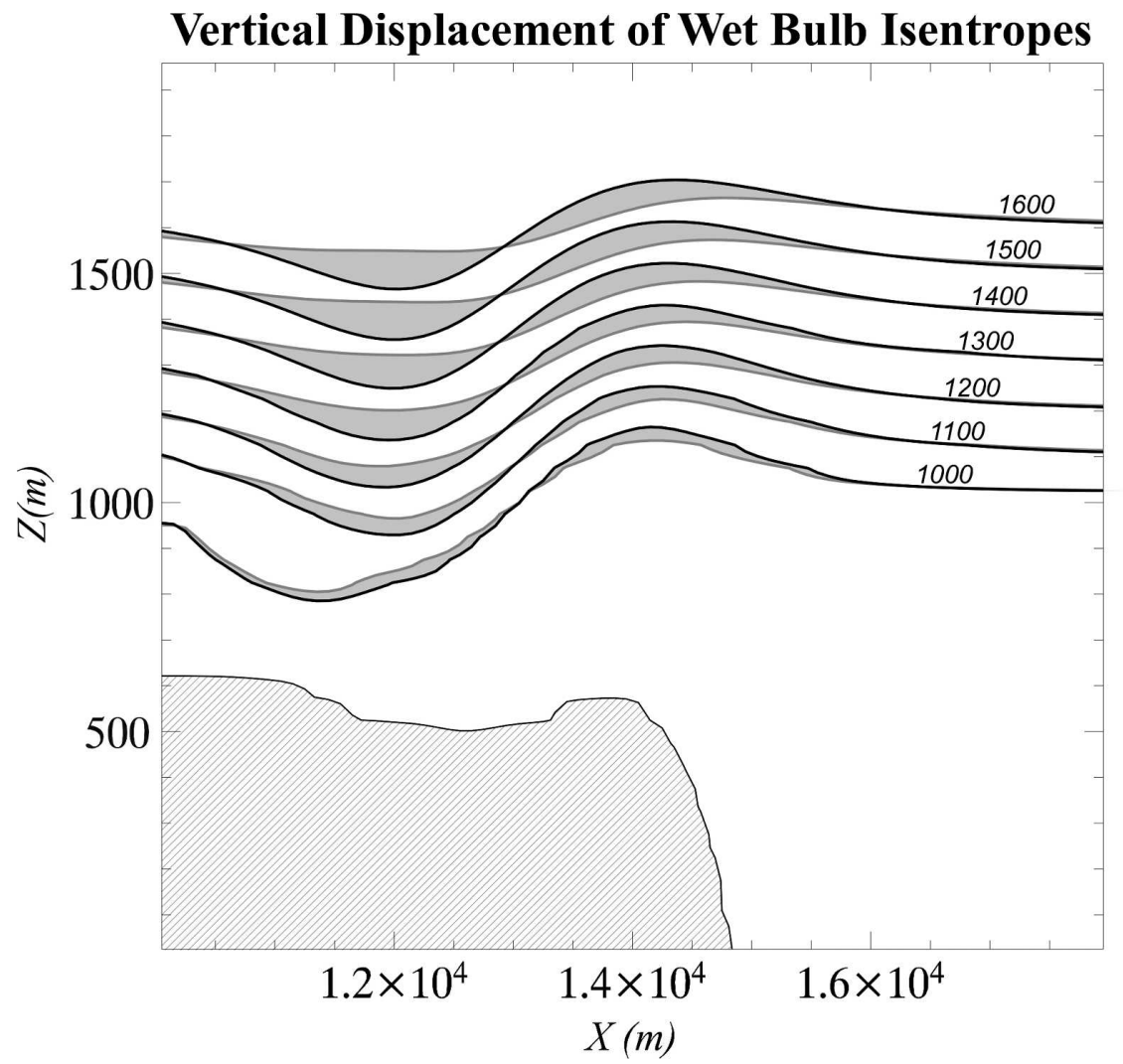

FIG. 8. Vertical displacement of wet-bulb potential temperature $\left(\theta_{w}\right)$ surfaces by forced ascent in the $\mathrm{d} \theta \mathrm{dz} 0$ and $\mathrm{d} \theta \mathrm{dz} 5$ experiments at $360 \mathrm{~s}$. The initial heights $(\mathrm{m})$ of each surface are indicated with italicized numbers. The surfaces for $\mathrm{d} \theta \mathrm{dz} 0(\mathrm{~d} \theta \mathrm{dz} 5)$ appear as black (gray) curves. The gray envelopes represent the regions within which the corresponding surfaces for the remaining experiments reside. The hatched region represents the density current for the $\mathrm{d} \theta \mathrm{dz} 0$ experiment.

est differences in upward motion between the $\mathrm{d} \theta \mathrm{dz} 0$ and $\mathrm{d} \theta \mathrm{dz} 5$ experiments (Fig. 10) appear in the 15002000-m layer in the presence of unsaturated ascent. Within this layer, the negative buoyancy force for unsaturated motions will be smaller in magnitude for larger lapse rates. Thus, the differences in forced ascent are directly attributable to differences in the ACBL lapse rates between experiments.

The foregoing analysis reveals that, even though density current depths vary little between experiments, the magnitude/depth of the forced ascent is clearly positively correlated with the lapse rate of the cloudbearing layer. But were these differences in the forced ascent sufficient to explain the failure of DCI in the three smallest lapse rate environments? If they were sufficient, then DCI should be prevented in the (large lapse rate) experiments that previously supported DCI by simply replacing the forced ascent in these experiments with the smaller forced ascent of the (small lapse rate) experiments that did not support DCI. While it is impossible to conduct these proposed experiments in a numerical modeling framework, the results of such experiments can be estimated by comparing two quantities: 1) the vertical displacement $(\Delta z)$ of each of the seven $\theta_{w}$ surfaces considered previously (Table 3 ) for the $\mathrm{d} \theta \mathrm{dz} 4$ and $\mathrm{d} \theta \mathrm{dz} 5$ experiments (at $7200 \mathrm{~s}$ ) and 2) the distance each representative $\theta_{w}$ surface of the $\mathrm{d} \theta \mathrm{dz} 0$, $\mathrm{d} \theta \mathrm{dz} 1$, and $\mathrm{d} \theta \mathrm{dz} 2$ environments would have to be vertically displaced from their reference heights to release thermal instability (i.e., $\Delta z_{k}^{*}$ for,

$$
\Delta z_{k}^{*}=\mathrm{LFC}_{Z_{k}}-Z_{k}
$$

where $Z_{k}$ is the $k$ th reference height and $\mathrm{LFC}_{Z_{k}}$ is the LFC for parcels lifted from $Z_{k}$ ). Experiments d $\theta \mathrm{dz} 4$ and $\mathrm{d} \theta \mathrm{dz} 5$ are used for $\Delta z$ because they not only failed to produce deep convection but also failed to release thermal instability through 7200 s. These quantities are plotted in Fig. 11 and illustrate that the vertical displacement of the isentropic levels originating between 


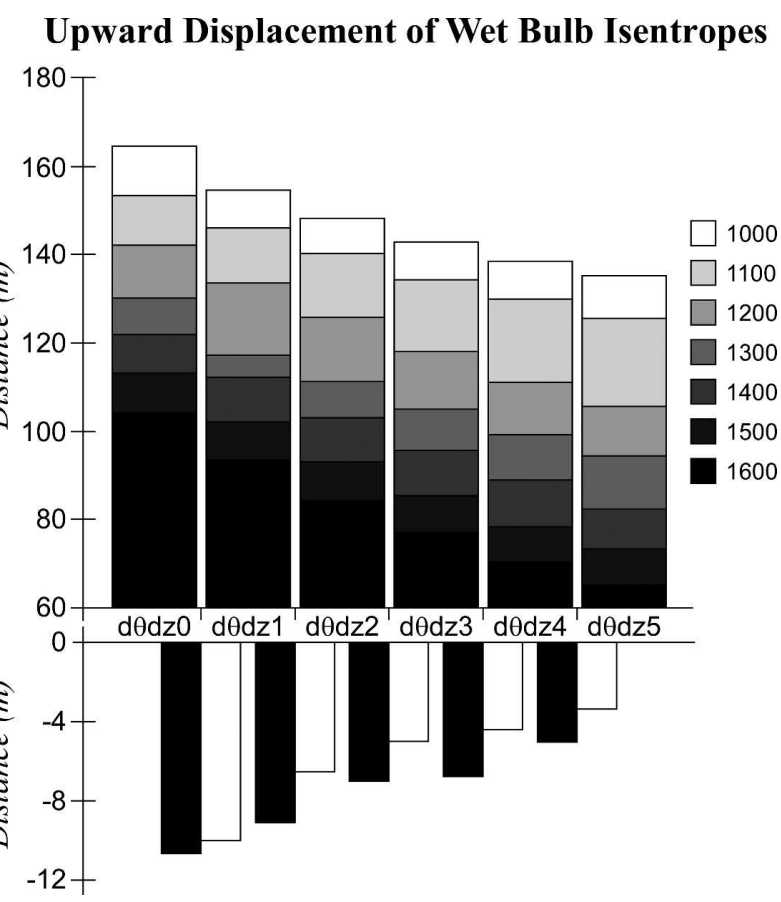

FIG. 9. (top) Upward displacement (in $\mathrm{m}$ from the reference height) of $\theta_{w}$ surfaces by forced ascent in the six experiments at 360 s. (bottom) Interexperiment differences in vertical displacement for the $\theta_{w}$ surfaces originating at 1000 and $1600 \mathrm{~m}$.

$\sim 1200$ and $\sim 1400 \mathrm{~m}$ in the $\mathrm{d} \theta \mathrm{dz} 4$ and $\mathrm{d} \theta \mathrm{dz} 5$ experiments exceeds the displacements necessary to release thermal instability in the $\mathrm{d} \theta \mathrm{dz} 0$ and $\mathrm{d} \theta \mathrm{dz} 1$ experiments $\left(\Delta z>\Delta z_{k}^{*}\right.$ for $\left.1400>k>1200\right)$. Thus, replacing the larger displacements of the $\mathrm{d} \theta \mathrm{dz} 0$ and $\mathrm{d} \theta \mathrm{dz} 1$ experiments with the smaller displacements of the $\mathrm{d} \theta \mathrm{dz} 4$ and $\mathrm{d} \theta \mathrm{dz} 5$ experiments would still have likely released thermal instability (the weaker forced ascent was still sufficient to lift parcels to the LFC) and would have probably still yielded DCI. Therefore, the sensitivity of forced ascent to the ACBL lapse rate is not a sufficient explanation for the sensitivity of DCI to the ACBL lapse rate.

While not a sufficient explanation, the sensitivity of forced ascent to the ACBL lapse rate cannot be neglected when taking full account of the sensitivity of DCI to the ACBL lapse rate. For example, as illustrated in Fig. 11, $\Delta z<\Delta z_{k}^{*}$ for the d $\theta \mathrm{dz} 2$ experiment; thus, reducing the upward displacement would have presumably prevented DCI in this experiment.

\section{2) INTEREXPERIMENT VARIATIONS IN PARCEL LFC}

As discussed in section 1, the precise nature of the sensitivity of parcel LFCs to the ACBL lapse rate will depend on the source of air within the convective clouds. If the source of air resides in the lowest $1000 \mathrm{~m}$ in these experiments, then $\theta_{w}$ dilution within cloudy air is necessary to realize interexperiment differences in the LFC. However, if the source or air resides above $1000 \mathrm{~m}$ in these experiments, the LFC will be different even in the absence of dilution.

To identify the source of air within the simulated deep convection, collections of weightless trajectories were initiated within the cloudy and positively buoyant air at 60-s intervals during the first 20 min after thermal instability was first released. These trajectories were then backward integrated to the beginning of the simulations using fourth-order Runge-Kutta temporal dif-

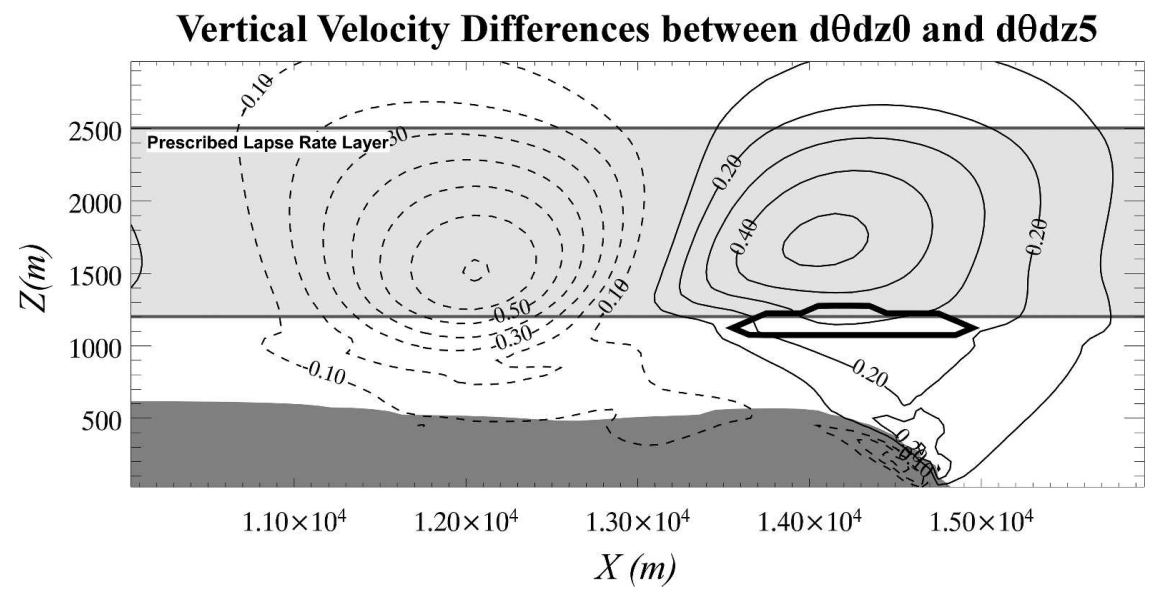

FIG. 10. Difference between the vertical velocity values of the $d \theta d z 0$ and $d \theta d z 5$ experiments at $360 \mathrm{~s}$. Thin black contours represent $w_{\mathrm{d} \theta \mathrm{d} z 0}-w_{\mathrm{d} \theta \mathrm{d} z 5}$ contoured every $0.1 \mathrm{~m} \mathrm{~s}^{-1}$ (broken contours are for values less than zero). Gray shaded region at the lower boundary represents the cold air behind the preexisting boundary of the $\mathrm{d} \theta \mathrm{dz} 0$ experiment. The cloud from the $\mathrm{d} \theta \mathrm{dz} 0$ experiment is indicated by the thick black curve. 


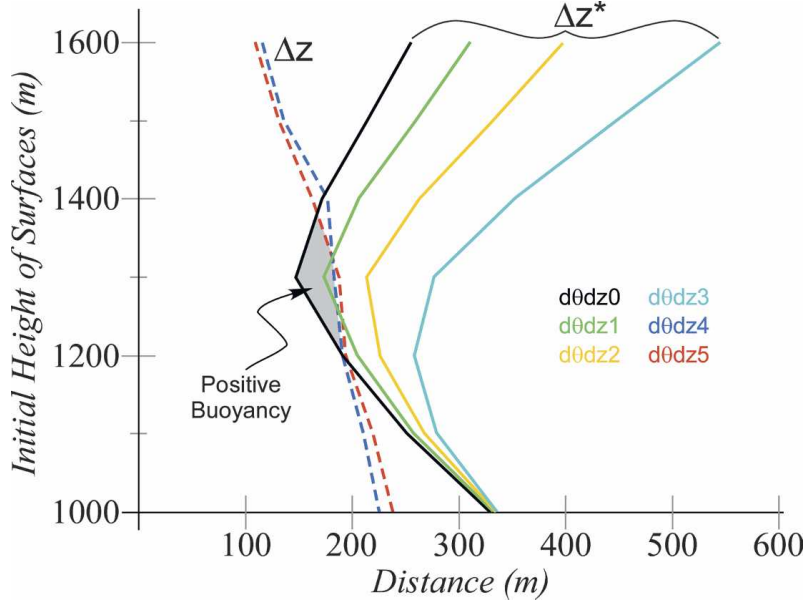

FIG. 11. Comparison of the vertical displacement of the wetbulb isentropic surfaces in the $\mathrm{d} \theta \mathrm{dz} 4$ and $\mathrm{d} \theta \mathrm{dz} 5$ experiments $(\Delta z)$ to the distance each representative wet-bulb isentropic surface of the $d \theta d z 0, d \theta d z 1$, and $d \theta d z 2$ environments would have to be vertically displaced from their reference heights to release thermal instability $\left(\Delta z^{*}\right)$.

ferencing at a primary time step of $15 \mathrm{~s}$ and an intermediate time step of $5 \mathrm{~s}$. Histograms of source regions were then synthesized for each of the experiments that yielded DCI. Histograms for the d $\theta \mathrm{dz} 0, \mathrm{~d} \theta \mathrm{dz} 1$, and d $\theta$ dz2 experiments appear in Fig. 12.

In all three experiments, deep convection was initiated from parcels originating above $1000 \mathrm{~m}$. The peak contribution came from parcels originating near 1300 $\mathrm{m}$. In fact, for the first 420,840 , and $1740 \mathrm{~s}$ of positive buoyancy for the $\mathrm{d} \theta \mathrm{dz} 0, \mathrm{~d} \theta \mathrm{dz} 1$, and $\mathrm{d} \theta \mathrm{dz} 2$ experiments, respectively, no parcels in the cloudy regions of positive buoyancy originated below $1000 \mathrm{~m}$. Parcels that originated below $1000 \mathrm{~m}$ in the early stages of the convective clouds and managed to ascend above their LCL, passed through the cloudy region at and behind the preexisting boundary, underneath the region of positive buoyancy, and became diluted before thermal instability could be released. However, as the convective cloud matured and upward motion increased, some of these saturated parcels were drawn into the developing cloud and became buoyant. This behavior manifested as a split flow and is illustrated in Fig. 13. Parcels that originated above $1000 \mathrm{~m}$ (green trajectories in Fig. 13) ascended within the buoyant cloud while parcels below $1000 \mathrm{~m}$ (blue trajectories in Fig. 13) followed flat if not descending tracks after passing over the preexisting boundary but were then ingested into the cloud.

This pattern of convective initiation is very similar to the DCI simulated by Fovell (2005). In his simulations of horizontal convective rolls and sea-breeze fronts, the first release of thermal instability occurred in parcels originated above the mixed layer. Parcels originating within the mixed layer contributed to the cloud only later when the convection was more fully developed.

If the simulated forced ascent was larger at $1000 \mathrm{~m}$ than it was above this level (Fig. 9), why is thermal instability released first in parcels originating above $1300 \mathrm{~m}$ ? Note from Fig. 11 that the value of $\Delta z^{*}$ was smallest at $1300 \mathrm{~m}$ in the three experiments that produced deep convection. Thus, while the upward displacement was larger at (and presumably below) 1000 $\mathrm{m}$, the vertical displacement that was necessary for the release of thermal instability was actually smaller in the $\sim 600 \mathrm{~m}$ above this point and was smallest at $1300 \mathrm{~m}$, the level from which the largest percentage of buoyant parcels originated.

It is important to reiterate that, despite the presence of a surface-based boundary, deep convection was initiated from elevated sources. The Glossary of Meteorology defines "elevated convection" as "convection that originates from an atmospheric layer above the boundary layer." While the convective clouds of these
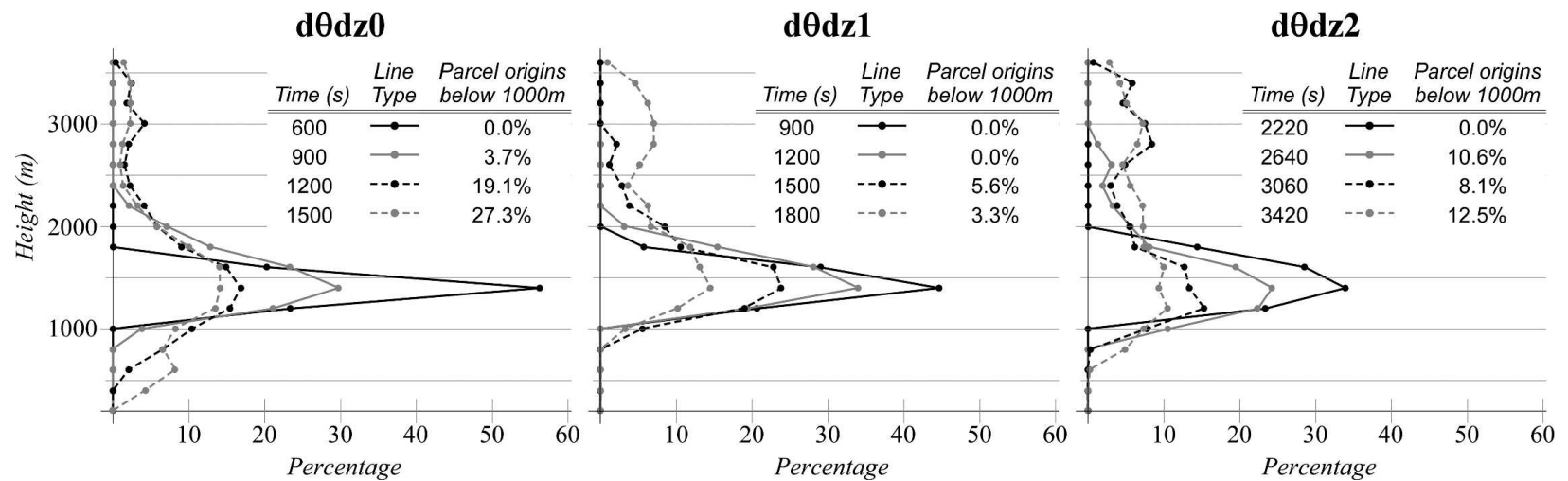

FIG. 12. Histograms of parcel sources for experiments d $\theta \mathrm{dz} 0, \mathrm{~d} \theta \mathrm{dz} 1$, and $\mathrm{d} \theta \mathrm{dz2}$. Height bins are in 200-m increments. Percentage below $1000 \mathrm{~m}$ is computed for the parcels originating between 0 and $2500 \mathrm{~m}$. 


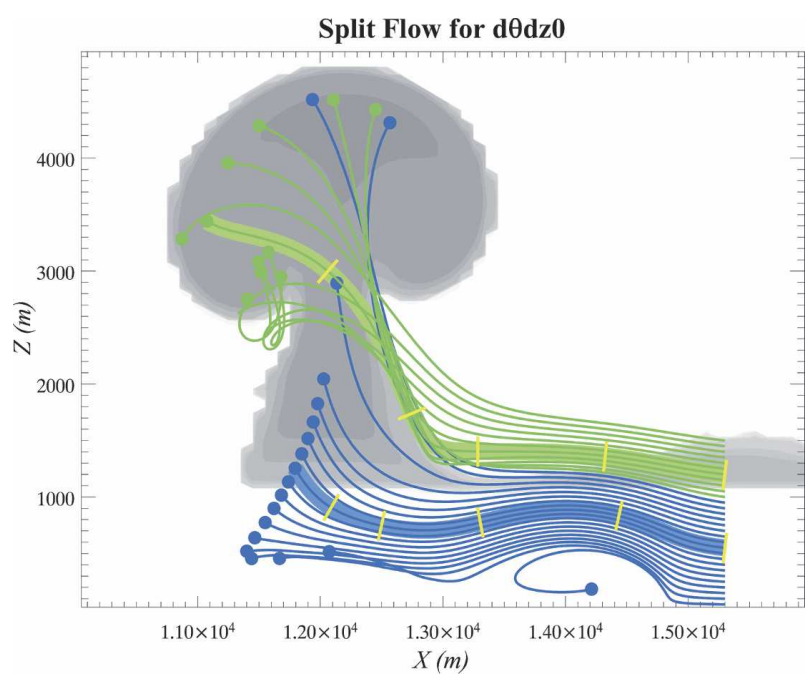

FIG. 13. Cloud field at $1200 \mathrm{~s}$ and trajectories forward integrated from $240 \mathrm{~s}$ for the $\mathrm{d} \theta \mathrm{dz} 0$ experiment. Trajectories originating above (below) $1000 \mathrm{~m}$ appear in green (blue). Trajectory positions at $1200 \mathrm{~s}$ appear as filled circles. The thick curves are representative trajectories in the two airstreams. Hatches along these representative trajectories are the trajectory positions every $192 \mathrm{~s}$.

experiments did initiate from sources above the wellmixed layer below $1000 \mathrm{~m}$, to describe them as elevated convection would be misleading since there was a significant contribution from low-level sources following initiation. The term elevated convection is also often associated with convective clouds with cloud bases above the ML-LCL, and while this is an informal definition only, it is not the case in these simulations since the active portions of the nascent deep convective clouds at the time of initiation surmount a forced cloud with a base at the ML-LCL (Fig. 14).
Based on the relationship between parcel LFC and ACBL lapse rates offered in section 1, the fact that simulated deep convection was initiated from elevated sources means that interexperiment differences in parcel LFCs that led to DCI failure in experiments d $\theta$ dz 4 and $\operatorname{d} \theta \mathrm{dz} 5$ (for which no parcel ascended above its LFC) did not require dilution, as would have been the case if convective clouds were initiated from air in the lowest $1000 \mathrm{~m}$. Instead, as illustrated in Fig. 15, the LFCs for elevated sources differ strictly as a result of differences in the ACBL lapse rates: the LFCs are lower in the larger ACBL lapse rate environments for air lifted from between 1000 and $\sim 2000 \mathrm{~m}$.

LFCs for elevated sources are clearly a function of the ACBL lapse rates but are these differences a sufficient explanation for the observed sensitivity of DCI to lapse rate? Based on the thought experiment of section $3 \mathrm{a}(1)$, forced ascent alone could not explain the sensitivity of DCI to the ACBL lapse rate but, given that $\Delta z<\Delta z_{k}^{*}$ for the $\mathrm{d} \theta \mathrm{d} z 2$ experiment (see Fig. 11), neither could it be neglected. Therefore, air parcels were more likely to reach their LFCs in the experiments with larger ACBL lapse rates because the forced ascent at the preexisting boundary was stronger and because the parcels' LFCs were lower, irrespective of parcel dilution.

\section{b. DCI failure in the presence of thermal instability release}

Like the $d \theta d z 4$ and $d \theta d z 5$ experiments, the $d \theta d z 3$ experiment failed to support DCI through $7200 \mathrm{~s}$ of simulation time following the introduction of the "seed." However, unlike $\mathrm{d} \theta \mathrm{dz} 4$ and $\mathrm{d} \theta \mathrm{dz} 5$, the $\mathrm{d} \theta \mathrm{dz} 3$ experiment did release thermal instability (Fig. 16). The

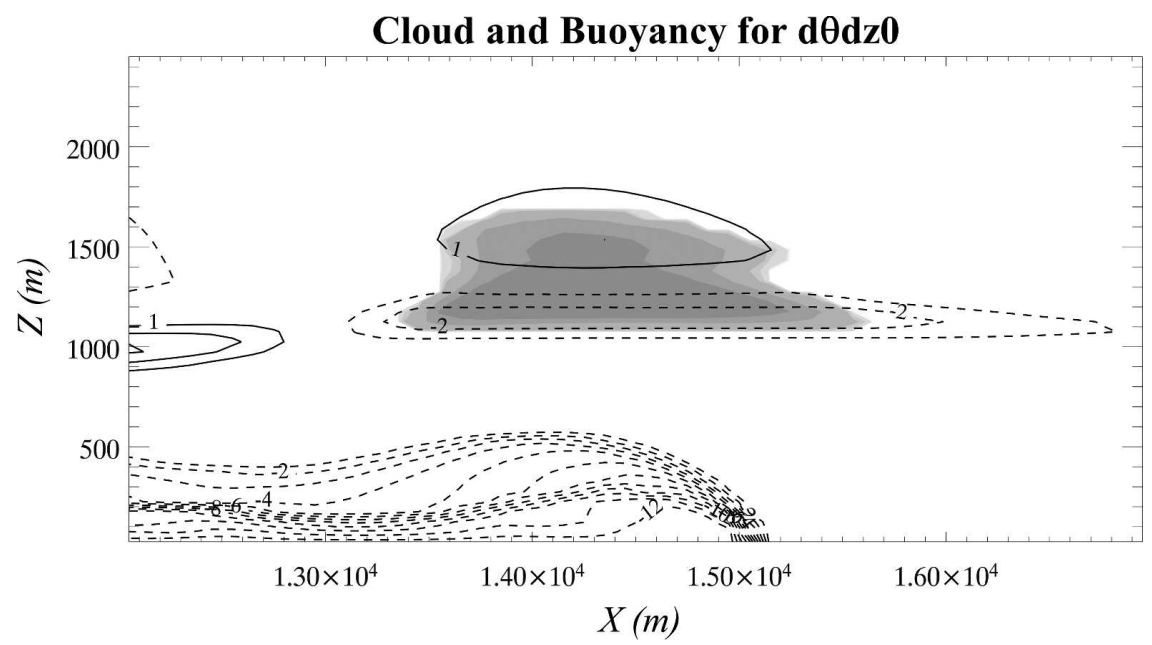

FIG. 14. Cloud and buoyancy for $\mathrm{d} \theta \mathrm{dz} 0$. Total cloud (cloud ice plus cloud water) is shaded at a logarithmic interval of $5 \times 10^{-n} \mathrm{~kg} \mathrm{~kg}^{-1}$ from a minimum value of $5 \times 10^{-7} \mathrm{~kg} \mathrm{~kg}^{-1}$. Buoyancy is contoured every $1 \times 10^{-2} \mathrm{~m} \mathrm{~s}^{-2}$ (negative values appear as broken contours). 


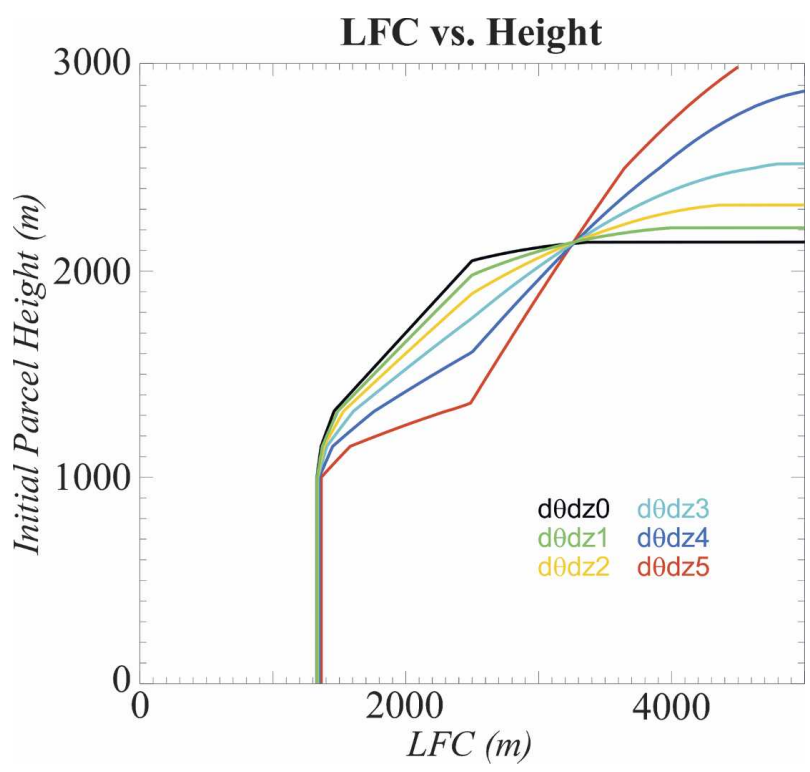

FIG. 15. LFC as a function of the height from which air is lifted for each of the environments used in these experiments.

resultant shallow convective clouds became diluted before producing precipitation. Thus, while DCI failure in the $\mathrm{d} \theta \mathrm{dz} 4$ and $\mathrm{d} \theta \mathrm{dz} 5$ experiments could be attributed to the failure of parcels to ascend to their LFCs, a similar explanation cannot be used to explain DCI failure in the $\mathrm{d} \theta \mathrm{dz} 3$ experiment.

Environments with constant lapse rates, as in the prescribed lapse rate layers used in these experiments, are examples of classic thermal instability wherein the vertical displacement produced by positive (negative) buoyancy acts, nonlinearly, to increase (decrease) the buoyancy and yield larger vertical displacement. However, if the buoyancy is reduced or the buoyant acceleration is mitigated by processes that increase the magnitude of the downward-directed pressure gradient force, then upward parcel displacement will be suppressed and it may not be possible to fully realize the thermal instability. In the absence of significant precipitation and other mitigating forces, changes in buoyancy due to parcel mixing/dilution will have be the most significant influence on regulating the full release of the thermal instability.

The maximum values of positive buoyancy within the primary simulated convective clouds (indicated with arrows in Fig. 16) are illustrated in Fig. 17 and clearly indicate the disparity in positive buoyancy between the $\mathrm{d} \theta \mathrm{dz} 2$ and $\mathrm{d} \theta \mathrm{dz} 3$ experiments. (The $\mathrm{d} \theta \mathrm{dz} 2$ experiment represents the experiment with the smallest ACBL lapse rate that still yielded DCI.) The time series in Fig. 17 correspond to the time period when the clouds reside within the prescribed lapse rate layer (between
1320 and $2500 \mathrm{~m}$ ). Both time series exhibit a nearly linear increase in buoyancy within the first $\sim 150 \mathrm{~s}$ but while this linear increase in buoyancy persists for the next $\sim 150 \mathrm{~s}$ in the $\mathrm{d} \theta \mathrm{dz} 3$ experiment, a nearly exponential increase in buoyancy occurs in the $\mathrm{d} \theta \mathrm{dz} 2$ experiment. Given that the environment of the $\mathrm{d} \theta \mathrm{dz} 2$ experiment is characterized by a larger ACBL lapse rate, it is not surprising to see the buoyancy increase more rapidly in this experiment than in the $d \theta d z 3$ experiment. However, a rapid increase in buoyancy never materializes in the $d \theta d z 3$ experiment and, thus, the full release of the thermal instability is being prevented.

Because a convective cloud is not a material surface, the intraexperiment changes and interexperiment differences in buoyancy noted above may not simply be the consequence of processes preventing the full release of thermal instability. Instead, the source of the air into the cloud may differ. Included in Fig. 17 are the mean heights of the source zones for the positively buoyant regions of the convective clouds in each experiment (computed using backward-integrated trajectories). The mean heights are consistently lower in the $\mathrm{d} \theta \mathrm{dz} 3$ experiment. A lower source height will mean a higher $\theta_{w}$ source but clearly this is not sufficient to yield larger buoyancy in the $\mathrm{d} \theta \mathrm{dz} 3$ experiment. It is also clear that there is no coherent trend in the mean heights for the $\mathrm{d} \theta \mathrm{dz} 3$ experiment. If the decrease in buoyancy was the result of a change to a higher average source (lower initial $\theta_{w}$ ), then the mean heights should systematically increase as the buoyancy decreases after $\sim 350 \mathrm{~s}$ of elapsed time. However, this trend is not apparent. Thus, the observed intraexperiment changes and interexperiment differences in buoyancy are not the result of changes in the source of air.

Because the changes in the source of air into the shallow convective cloud of the $\mathrm{d} \theta \mathrm{dz} 3$ experiment are unable to explain the failure of this cloud to evolve into deep convection, DCI failure must be attributable to processes that mitigate the full release of thermal instability. The preceding analysis suggests that DCI failure in experiment $\mathrm{d} \theta \mathrm{dz} 3$ is likely a consequence of a deficiency in positive buoyancy and thus that mixing/ dilution may be important. The fact that the buoyancy in experiment $\mathrm{d} \theta \mathrm{dz} 3$ increases linearly with time along with the apparent steady ascent of the convective cloud (Fig. 17) suggest that, initially, the buoyancy is large enough to support a trend toward deep convection. However, unlike in experiment $\mathrm{d} \theta \mathrm{dz} 2$, the rapid increase in buoyancy never appears and the buoyancy slowly decreases as the rate of convective cloud ascent slows. This suggests that mixing/dilution may be truncating the evolution toward deep convection in the $\mathrm{d} \theta \mathrm{dz} 3$ experiment. 

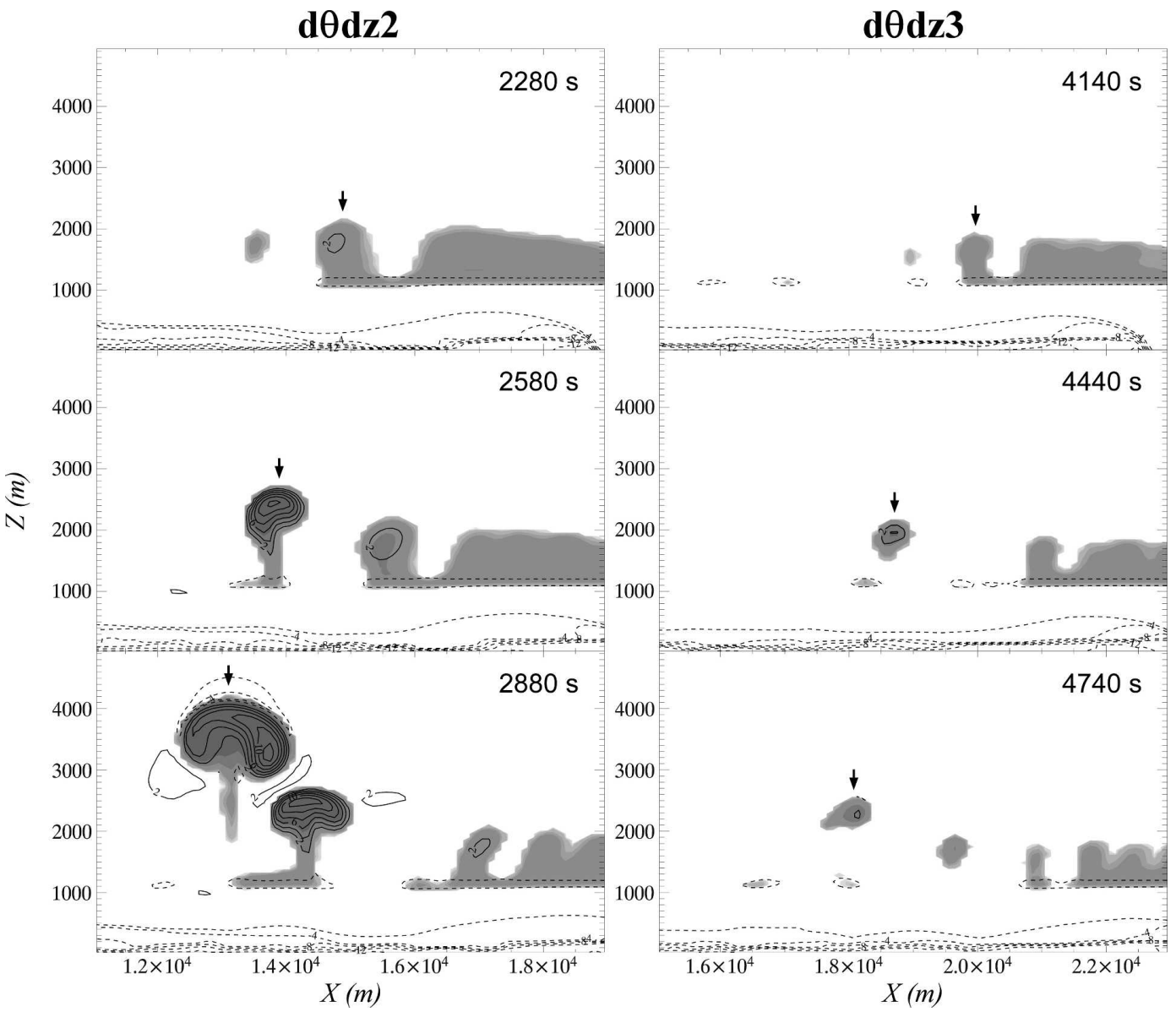

FIG. 16. Evolution of the primary convective clouds in the $d \theta d z 2$ and $d \theta d z 3$ experiments. Total cloud (cloud ice plus cloud water) is shaded at a logarithmic interval of $5 \times 10^{-n} \mathrm{~kg} \mathrm{~kg}^{-1}$ from a minimum value of $5 \times 10^{-7} \mathrm{~kg}$ $\mathrm{kg}^{-1}$. Buoyancy is contoured every $2 \times 10^{-2} \mathrm{~m} \mathrm{~s}^{-2}$ (negative values appear as broken contours). Arrows denote the primary convective cloud for each experiment.

The best way to quantify the dilution is to consider trajectories through the clouds. Trajectories were backward computed from the regions of the convective clouds with the highest positive buoyancy. Trajectories were then matched based on the source zone and termination altitude (because of the backward integration, this is actually the initial position). Values of $\theta_{w}$ plotted as a function of trajectory height for representative trajectories at three times appear in Fig. 18. The $\theta_{w}$ values are nearly identical early in the evolution of the convective clouds in each experiment (Fig. 18a) with only slightly more dilution in the $\mathrm{d} \theta \mathrm{dz} 3$ experiment. However, as the convective clouds continue to evolve, the dilution increases markedly in the $\mathrm{d} \theta \mathrm{dz} 3$ experiment, yielding $\theta_{w}$ values that are considerably smaller than those in the $\mathrm{d} \theta \mathrm{dz} 2$ experiment (Figs. 18b and 18c). The differences in dilution are in part attributable to the differences in the initial cloud size. As evidenced in Fig. 16 , the embryonic convective cloud in $\mathrm{d} \theta \mathrm{dz} 2$ at $5880 \mathrm{~s}$ was demonstrably larger (1.7 times larger) than the embryonic convective cloud in $\mathrm{d} \theta \mathrm{dz} 3$ at $7740 \mathrm{~s}$. Some of this difference may be a consequence of the larger area of positive buoyancy stemming from the larger ACBL lapse rate in the $\mathrm{d} \theta \mathrm{dz} 2$ experiment; however, a more important mechanism appears to be primarily responsible for the increased dilution of the convective cloud of the $d \theta d z 3$ experiment.

As indicated in Fig. 18, the interexperiment differences in the elapsed times required for the specific trajectories to ascend from $1350 \mathrm{~m}$ to the altitude of trajectory initiation (coincidentally $1350 \mathrm{~m}$ corresponds to both the approximate bottom of the prescribed lapse rate layer and the level at which the trajectories illustrated in Fig. 18 have the same $\theta_{w}$ ) change as the convective cloud evolves. Approximately $200 \mathrm{~s}$ after the shallow convective clouds cleave off of the cloud mass at the preexisting boundary, the trajectory of the $d \theta d z 3$ experiment takes about $15 \%$ longer to traverse the 


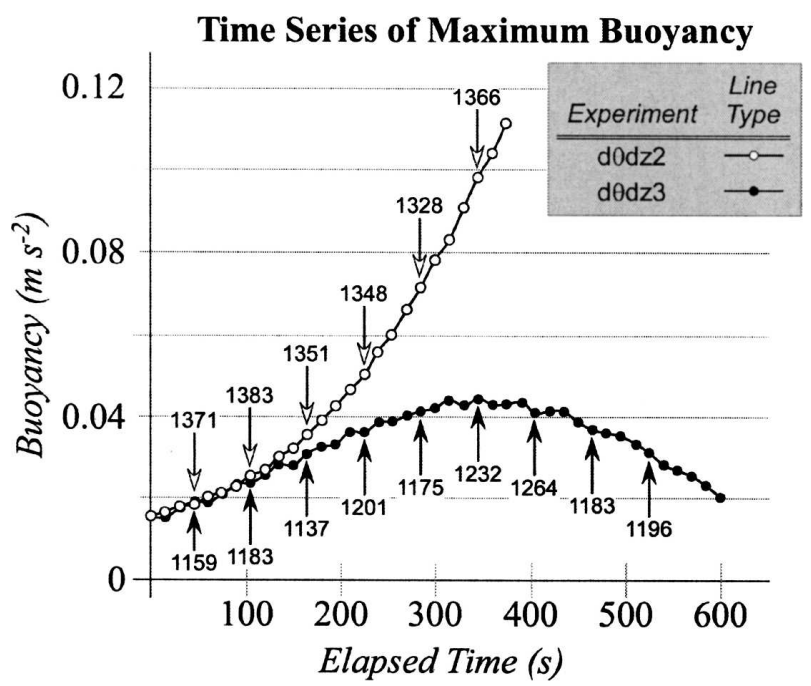

FIG. 17. Time series of maximum buoyancy within the primary clouds of the $d \theta d z 2$ and $d \theta d z 3$ experiments. Elapsed time is measured from $2175 \mathrm{~s}$ for $\mathrm{d} \theta \mathrm{dz} 2$ and from $4155 \mathrm{~s}$ for $\mathrm{d} \theta \mathrm{dz} 3$. Numbers atop (below) open (closed) arrows indicate the average source height for air within the positively buoyant cloud for the $\mathrm{d} \theta \mathrm{dz} 2$ $(\mathrm{d} \theta \mathrm{dz} 3)$ experiment at the given time.

same depth as the trajectory of the $\mathrm{d} \theta \mathrm{dz} 2$ experiment (Fig. 18a). The same is roughly true of the trajectories at the next times illustrated in Fig. 18b. However, for trajectories that ascend to $\sim 2300 \mathrm{~m}$ at $4620 \mathrm{~s}$ in $\mathrm{d} \theta \mathrm{dz} 3$ and $2580 \mathrm{~s}$ in $\mathrm{d} \theta \mathrm{dz} 2$ (Fig. 18c) a more prominent interexperiment disparity in the elapsed times emerges: the trajectories of the $\mathrm{d} \theta \mathrm{dz} 3$ experiment take considerably longer to ascend to the same altitude than the trajec- tories of the $\mathrm{d} \theta \mathrm{dz} 2$ experiment. To a large extent, this result should be expected considering that the environment of the $\mathrm{d} \theta \mathrm{dz} 3$ experiment has a smaller low-level CAPE density (the portion of the CAPE below a given level) for undiluted parcels originating just above 1000 m (Fig. 19). However, the larger buoyancy force may not be the only factor to consider since a slower trajectory ascent should yield a larger amount of dilution (even if the dilution rates for the trajectories of the two experiments are the same) and the reduction in buoyancy more pronounced. This reduction in buoyancy will lead to a slower ascent (compared to the less diluted result) and will further increase the dilution. Thus, the relationship between dilution and buoyancy/parcel ascent is nonlinear and suggests that two separate convective regimes may exist: a supercritical state capable of supporting DCI and a subcritical state that is unlikely to support DCI. It is important to note that dilution will act to reduce the buoyancy in both subcritical and supercritical conditions. However, in supercritical conditions, the rate of increase in buoyancy due to continued parcel ascent exceeds the reduction in buoyancy due to dilution. Under subcritical conditions, the rate of increase in buoyancy due to parcel ascent is outpaced by the rate of reduction in buoyancy due to dilution and the parcel ascent terminates.

The relationship between the environmental lapse rate and the two convective regimes (one capable of supporting DCI and the other unlikely to support DCI) is illustrated here using a heuristic Lagrangian model. In this model, 1) parcel motion is purely vertical, 2) parcel acceleration is solely dependent on buoyancy, 3 )

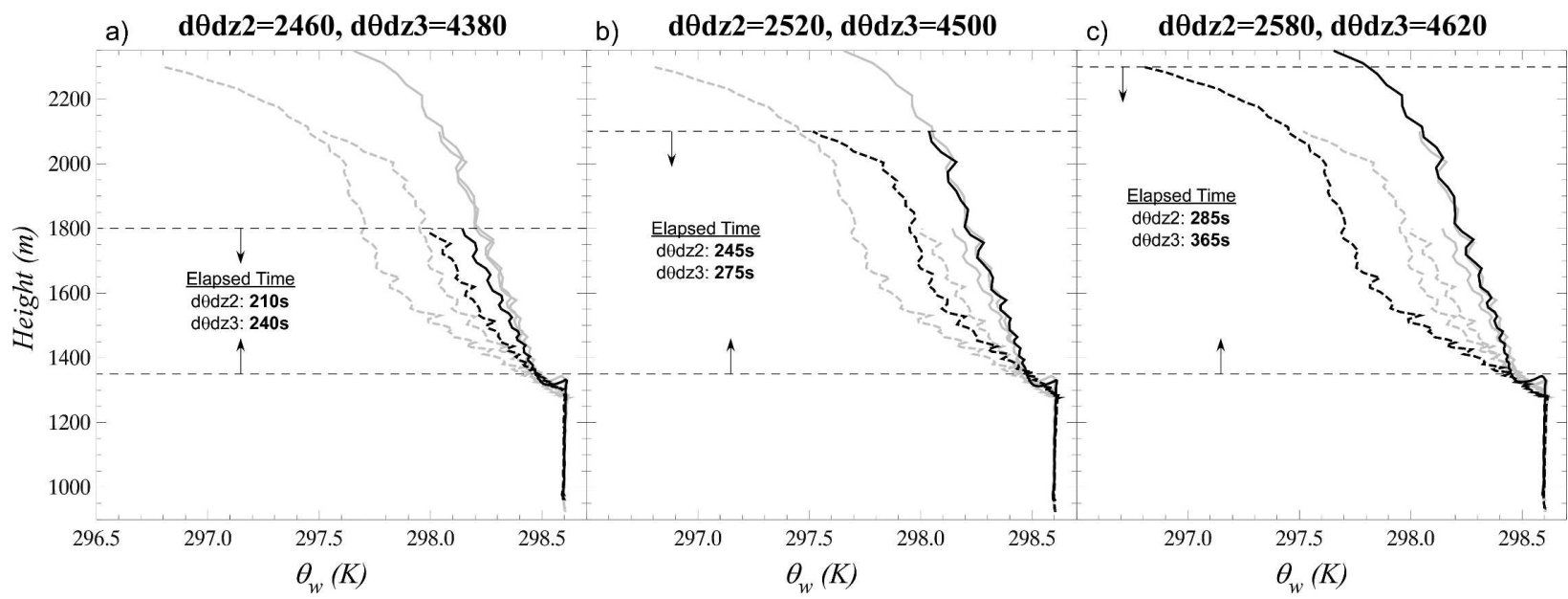

FIG. 18. Values of $\theta_{w}$ plotted as a function of height for representative trajectories from the $\mathrm{d} \theta \mathrm{dz2}$ (continuous curves) and d $\theta \mathrm{dz} 3$

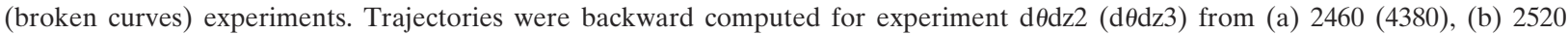
(4500), and (c) $2580 \mathrm{~s} \mathrm{(4620} \mathrm{s).} \mathrm{Included} \mathrm{in} \mathrm{these} \mathrm{panels} \mathrm{are} \mathrm{the} \mathrm{elapsed} \mathrm{times} \mathrm{necessary} \mathrm{for} \mathrm{the} \mathrm{trajectories} \mathrm{to} \mathrm{ascend} \mathrm{from} 1350 \mathrm{~m}$ to the termination altitude (because of the backward integration, this is actually the initial position). Gray curves are the profiles from the other panels. 


\section{CAPE Density vs. Height}

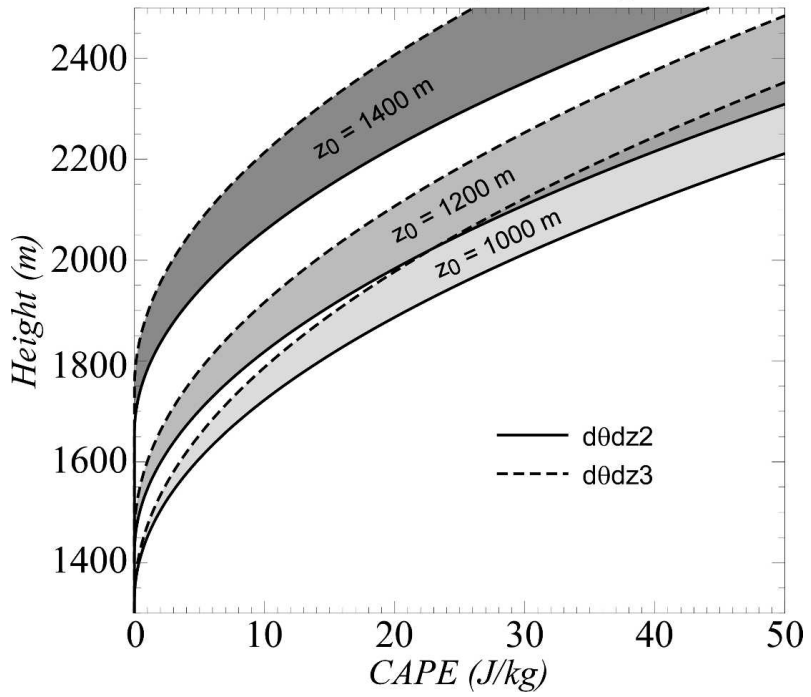

FIG. 19. CAPE density (the portion of the CAPE below a given level) for undiluted parcels plotted as a function of the height below which the CAPE density is computed. The continuous (broken) curves represent profiles for the $d \theta d z 2(d \theta d z 3)$ experiment. CAPE density is computed for three initial parcel heights $\left(z_{0}\right): 1000,1200$, and $1400 \mathrm{~m}$. For each $z_{0}$ the profiles for each experiment are visually coupled using the shaded envelopes.

the dilution rate (of $\theta_{w}$ ) is constant, ${ }^{3}$ and 4 ) in the absence of dilution, $\theta_{w}$ is conserved. The model is solved using the environmental profiles of temperature and moisture used for the experiments conducted for this work. Additional information on the model can be found in appendix B. Results appear in Fig. 20 and clearly illustrate the two regimes. For these conditions, the critical value of $\Gamma$ is $8.0 \mathrm{~K} \mathrm{~km}^{-1}$ (corresponding to $d \theta_{0} / d z=1.985 \mathrm{~K} \mathrm{~km}^{-1}$ ): values of $\Gamma$ larger than this constitute a supercritical state, would produce parcel ascent through the depth of the domain, and would presumably support DCI. This critical value should not be considered a "magic number" as it is highly sensitive to the choices of dilution rate and initial vertical motion. However, this model clearly illustrates the presence of two regimes for convective clouds that are regulated by the ACBL lapse rate and indicates that the failure of experiment $\mathrm{d} \theta \mathrm{dz} 3$ to yield deep convection is a consequence of the small ACBL lapse rate, which supported subcritical conditions.

\footnotetext{
${ }^{3}$ The validity of such an assumption is uncertain. As the positive buoyancy within a cloud increases, the entrainment should increase, suggesting that the dilution rate should be positively correlated with low-level CAPE density. Including this sensitivity in the heuristic model could shift the critical lapse rate to a smaller value but should not alter the ability of the model to capture the two regimes.
}

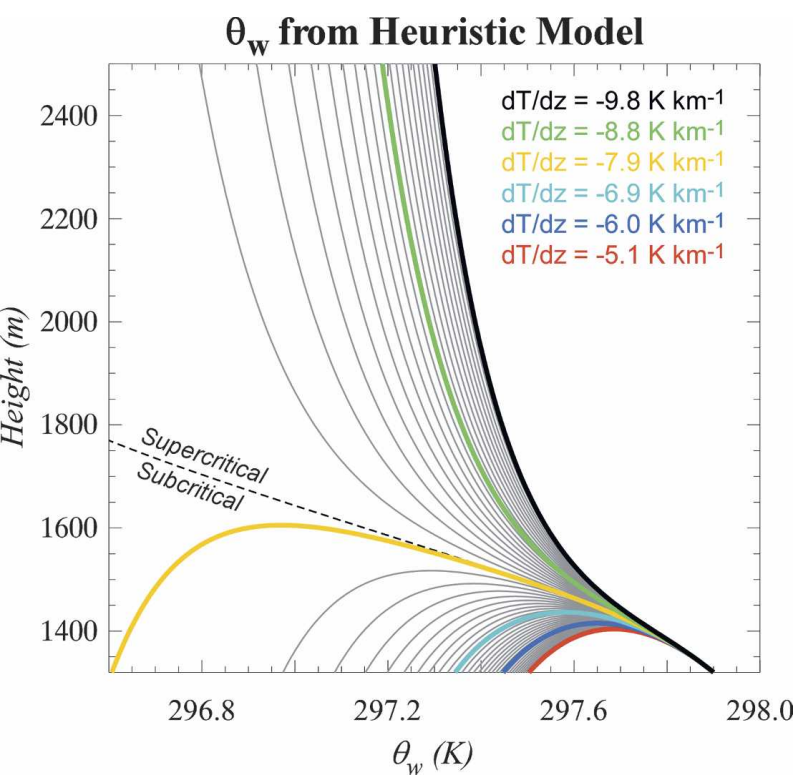

FIG. 20. Values of $\theta_{w}$ plotted as a function of height computed using the heuristic Lagrangian model. Thick curves are associated with the six ACBL lapse rates used in the experiments conducted for this work. Gray curves are for intermediate lapse rates.

\section{Conclusions}

The aim of this work was to examine the possible sensitivity of deep convective initiation to the lapse rate of the active cloud-bearing layer. A suite of numerical experiments was conducted using an idealized cloudscale model. Clouds were initiated using a new technique that involves a preexisting airmass boundary initialized such that the (unrealistic) adjustment of the model state variables to the imposed boundary is disassociated from the simulation of convection. The reference state environments had the same ML-CIN, MLCAPE, ML-LFC, relative humidity, and wind profiles but varying lapse rates in the ACBL (located between the ML-LFC and $2500 \mathrm{~m}$ ). The following is a summary of the findings from this work.

- Of the six simulations conducted for the experiment set, only the three environments with the largest ACBL lapse rates supported DCI.

- Among the environments that supported DCI, the time required for initiation decreased with increasing lapse rate and the magnitude of the maximum vertical velocity value for the initial deep convection was positively correlated with the lapse rate.

- Even though density current depths varied little between experiments, the magnitude/depth of the forced ascent increased with the increasing lapse rate of the cloud-bearing layer. This was a consequence of 
the smaller values of negative buoyancy within the ACBL for the larger lapse rate environments.

- Despite the presence of a surface-based airmass boundary, deep convection was initiated from elevated sources: parcels in the convective clouds originated near $1300 \mathrm{~m}$ and the contribution from air in the well-mixed layer (below $1000 \mathrm{~m}$ ) was delayed by as much as $1740 \mathrm{~s}$ following the first release of thermal instability. Parcels originated near $1300 \mathrm{~m}$ because the value of $\Delta z^{*}$ was smallest at this level. The quantity $\Delta z^{*}$ is the distance a parcel would have to be lifted from its reference height to reach its LFC.

- Thermal instability was more likely to be released in the experiments with larger ACBL lapse rates because the forced ascent at the preexisting boundary was stronger and because the parcels' LFCs were lower, irrespective of parcel dilution.

- A heuristic Lagrangian model was offered that captured the nonlinear relationship between buoyancy and dilution and clearly illustrated the presence of two regimes. In one regime (termed supercritical), the rate of increase in the buoyancy due to parcel ascent exceeds the reduction in the buoyancy due to dilution. DCI is likely in this regime. In the second regime (termed subcritical), the rate of increase in the buoyancy due to parcel ascent is outpaced by the rate of reduction in the buoyancy from dilution. DCI is unlikely in this regime. The transition from subcritical to supercritical conditions is strongly dependent on the environmental lapse rate.

- In experiment $\mathrm{d} \theta \mathrm{dz} 3$ parcels did reach their LFCs (thermal instability was released) but deep convection still failed to develop. It was argued that, unlike the experiments with larger ACBL lapse rates, the lapse rate of the $d \theta d z 3$ experiment yielded subcritical conditions.

This work has shown that the lapse rate of the ACBL will regulate the convective regime irrespective of boundary layer thermodynamics, deep tropospheric vertical shear, and forced ascent and should therefore be considered along with these parameters when attempting to diagnosis and/or predict DCI.

Acknowledgments. The authors wish to thank John Nielsen-Gammon, Matthew Parker, and an anonymous reviewer for their thorough reviews. Thanks also go to Conrad Ziegler and Roger A. Pielke Sr. for their comments in the early stages of this work. All simulations were conducted on the IBM SP at Purdue University's Rosen Center for Advanced Computing. Funding for this work was provided through NSF Grant ATM0233780 (Dr. S. Nelson) and NASA Grants THPNNG04GI84G (Dr. J. Entin) and IDS-NNG04GL61G (Drs. J. Entin and G. Gutman).

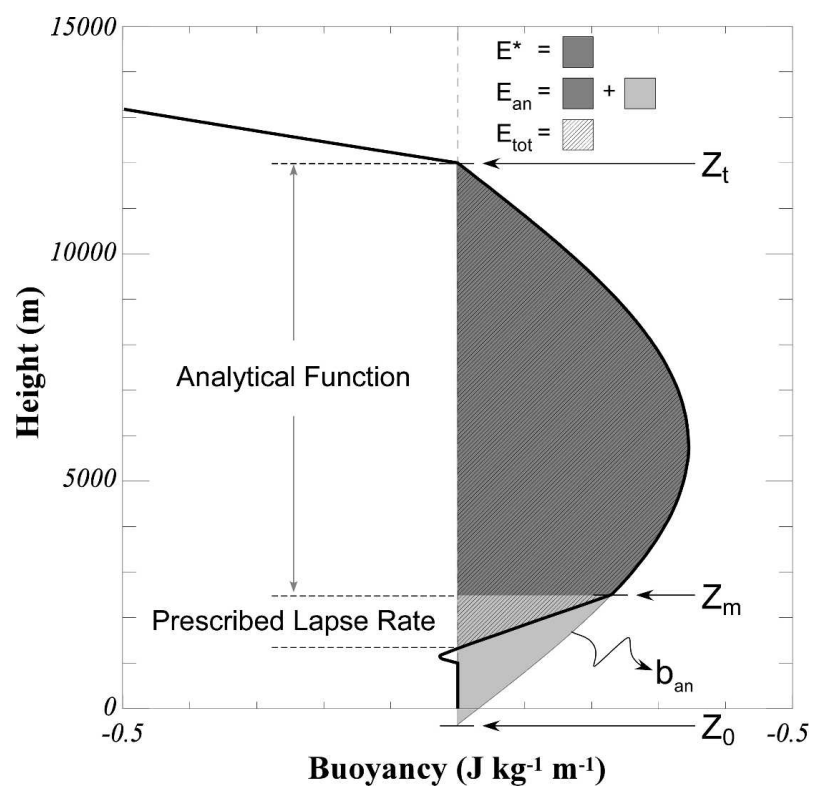

FIG. A1. Illustration of the analytical function used to prescribe the temperature profiles of the experiment soundings. The buoyancy distribution for the sounding appears as a continuous black curve, $z_{t}$ represents the height of the tropopause, $z_{0}$ represents the bottom of the full (untruncated) analytical sounding, $z_{m}$ represents the top of the prescribed lapse rate layer and bottom of the truncated analytical sounding, the entire shaded region represents the ML-CAPE of the full (untruncated) analytical buoyancy profile $\left(E_{\text {an }}\right)$, the dark shading represents the ML-CAPE of the truncated analytical buoyancy profile $\left(E^{*}\right)$, and the hatched region represents the ML-CAPE of the final sounding.

\section{APPENDIX A}

\section{Analytical Prescription of Reference State Temperature}

The reference state temperature profile is prescribed using an analytical function for buoyancy that relates the ML-CAPE within the prescribed lapse rate layer to the fixed-total ML-CAPE. The analytical function is as follows:

$$
b_{\text {an }}=\frac{\pi}{2\left(z_{t}-z_{0}\right)} E_{\text {an }} \sin \left[\frac{\pi\left(z-z_{0}\right)}{z_{t}-z_{0}}\right],
$$

where $b_{\text {an }}$ is the buoyancy $\left(\mathrm{J} \mathrm{kg}^{-1} \mathrm{~m}^{-1}\right)$. The relationship between the remaining parameters in (A1) is illustrated in Fig. A1 for an example sounding. The parameter $z_{t}$ is the height of the tropopause, $z_{0}$ is the bottom elevation of the full analytical buoyancy distribution, and $E_{\text {an }}$ is the ML-CAPE of the full (untruncated) analytical buoyancy profile (equivalent to $\int_{z_{0}}^{z_{t}} b_{\text {an }} d z$ ).

The magnitude of $E_{\mathrm{an}}$, and therefore the amplitude of the sine profile used in the analytical function, is used to modulate the shape of the buoyancy/temperature 
profile above the prescribed lapse rate layer. Because the total ML-CAPE of the final sounding $\left(E_{\text {tot }}\right)$ is to remain constant between reference state environments, the profile of the buoyancy/temperature above the prescribed lapse rate layer must be adjusted to account for the intersounding differences in the ML-CAPE within the prescribed lapse rate layer $\left(E_{\mathrm{plr}}\right)$. For a fixed $E_{\text {tot }}$, the CAPE of the truncated analytical profile, $E^{*}$ (the portion of the analytical profile above the prescribed lapse rate layer; see Fig. A1) must satisfy the following relation:

$$
E^{*}=E_{\mathrm{tot}}-E_{\mathrm{plr}} .
$$

With this definition of $E^{*}, E_{\text {an }}$ can be written as

$$
\begin{aligned}
E_{\mathrm{an}} & =E^{*}+\int_{0}^{z_{m}} b_{\mathrm{an}} d z \\
& =E^{*}\left\langle 1+\frac{1}{2}\left\{\cos \left[\frac{\pi\left(z_{m}-z_{0}\right)}{z_{t}-z_{0}}\right]-1\right\}\right\rangle^{-1},
\end{aligned}
$$

where $z_{m}$ is the height of the top of the prescribed lapse rate layer. This equation still contains one unknown, $z_{0}$; however, only one value of $z_{0}$ will simultaneously satisfy (A3) while ensuring that the analytical buoyancy at the top of the prescribed lapse rate layer equals the prescribed buoyancy.

\section{APPENDIX B}

\section{Heuristic Lagrangian Model}

Four key assumptions underpin the heuristic Lagrangian model used for this work. The first assumption is that parcel motion is purely vertical. The second assumption is that parcel acceleration is solely dependent on the buoyancy. In this model, buoyancy accounts for the effect of water vapor on density but excludes precipitation loading. The buoyancy is computed using the diluted $\theta_{w}$ within the parcel and the environmental profiles of temperature and moisture used for the DCI experiments. The third assumption is that the dilution rate is constant. Only the wet-bulb potential temperature is diluted and the dilution rate has been set to $0.002 \mathrm{~K} \mathrm{~s}^{-1}$. This dilution rate is consistent with the values exhibited by trajectories backward computed from the regions of peak buoyancy within the convective clouds of the $d \theta d z 2$ and $d \theta d z 3$ experiments. The fourth key assumption is that $\theta_{w}$ is conserved in the absence of dilution. This model also assumes that the parcel pressure is always identical to the environmental (hydrostatic) pressure.

Parcels are initialized identically, regardless of the environmental profile. It is assumed that the source
TABLE B1. Prescribed parameters in the heuristic Lagrangian model and the specific values used for the experiments conducted.

\begin{tabular}{lc}
\hline \multicolumn{1}{c}{ Parameter } & Value \\
\hline Initial $\theta_{w}$ & $297.9 \mathrm{~K}$ \\
Initial height & $1320 \mathrm{~m}$ \\
Initial vertical velocity & $1.5 \mathrm{~m} \mathrm{~s}^{-1}$ \\
Dilution rate & $0.002 \mathrm{~K} \mathrm{~s}^{-1}$ \\
\hline
\end{tabular}

height of the parcels is $\sim 1320 \mathrm{~m}$. At this height, the environmental $\theta_{w}$ values are identical between the different environments and have a value of $297.9 \mathrm{~K}$. Parcels are also given an initial upward motion of $1.5 \mathrm{~m} \mathrm{~s}^{-1}$. A summary of the prescribed parameters appears in Table B1.

\section{REFERENCES}

Atkins, N. T., M. L. Weisman, and L. J. Wicker, 1999: The influence of preexisting boundaries on supercell evolution. Mon. Wea. Rev., 127, 2910-2927.

Bryan, G. H., 2005: Spurious convective organization in simulated squall lines owing to moist absolutely unstable layers. Mon. Wea. Rev., 133, 1978-1997.

_ , and J. M. Fritch, 2002: A benchmark simulation for moist nonhydrostatic numerical models. Mon. Wea. Rev., 130, 2917-2928.

Coniglio, M. C., D. J. Stensrud, and L. J. Wicker, 2006: Effects of upper-level shear on the structure and maintenance of strong quasi-linear mesoscale convective systems. J. Atmos. Sci., 63, 1231-1252.

Dudhia, J., and M. W. Moncrieff, 1989: A three-dimensional numerical study of an Oklahoma squall line containing rightflank supercells. J. Atmos. Sci., 46, 3363-3391.

Fovell, R. G., 2005: Convective initiation ahead of the sea-breeze front. Mon. Wea. Rev., 133, 264-278.

, and P. S. Dailey, 1995: The temporal behavior of numerically simulated multicell-type storms. Part I: Modes of behavior. J. Atmos. Sci., 52, 2073-2095.

_, and P.-H. Tan, 1998: The temporal behavior of numerically simulated multicell-type storms. Part II: The convective cell life cycle and cell regeneration. Mon. Wea. Rev., 126, 551577

—, and —, 2000: A simplified squall-line model revisited. Quart. J. Roy. Meteor. Soc., 126, 173-188.

Garner, S. T., and A. J. Thorpe, 1992: The development of organized convection in a simplified squall-line model. Quart. J. Roy. Meteor. Soc., 118, 101-124.

Gilmore, M. S., and L. J. Wicker, 1998: The influence of midtropospheric dryness on supercell morphology and evolution. Mon. Wea. Rev., 126, 943-958.

_ J. M. Straka, and E. N. Rasmussen, 2004a: Precipitation evolution sensitivity in simulated deep convective storms: Comparisons between liquid-only and simple ice and liquid phase microphysics. Mon. Wea. Rev., 132, 1897-1916.

,-- , and,$- 2004 \mathrm{~b}$ : Precipitation uncertainty due to variations in precipitation particle parameters within a simple microphysics scheme. Mon. Wea. Rev., 132, 2610-2627. 
Hane, C. E., 1975: The trajectories of dropsondes in simulated thunderstorm circulations. Mon. Wea. Rev., 103, 709-716.

Houston, A. L., 2004: The role of preexisting airmass boundaries in the maintenance and rotation of deep convection in a highCAPE, low-shear environment. Ph.D. thesis, Dept. of Atmospheric Sciences, University of Illinois at UrbanaChampaign, 275 pp.

Johnson, R. H., and B. E. Mapes, 2001: Mesoscale processes and severe convective weather. Severe Convective Storms, $\mathrm{Me}$ teor. Monogr., No. 50, Amer. Meteor. Soc., 71-122.

Klemp, J. B., and R. B. Wilhelmson, 1978: The simulation of three-dimensional convective storm dynamics. J. Atmos. Sci., 35, 1070-1096.

Lee, B. D., and R. B. Wilhelmson, 1997: The numerical simulation of nonsupercell tornadogenesis. Part II: Evolution of a family of tornadoes along a weak outflow boundary. J. Atmos. Sci., 54, 2387-2415.

Lin, Y.-L., R. L. Deal, and M. S. Kulie, 1998: Mechanisms of cell regeneration, development, and propagation within a twodimensional multicell storm. J. Atmos. Sci., 55, 1867-1886.

Liu, C., and M. W. Moncrieff, 1996: A numerical study of the effects of ambient flow and shear on density currents. Mon. Wea. Rev., 124, 2282-2303.

— and - 2000: Simulated density currents in idealized stratified environments. Mon. Wea. Rev., 128, 1420-1437.

Murray, F. W., 1970: Numerical models of a tropical cumulus cloud with bilateral and axial symmetry. Mon. Wea. Rev., 98, 14-28.

Ogura, Y., 1963: The evolution of a moist convective element in a shallow, conditionally unstable atmosphere: A numerical calculation. J. Atmos. Sci., 20, 407-424.

Peckham, S. E., and L. J. Wicker, 2000: The influence of topography and lower-tropospheric winds on dryline morphology. Mon. Wea. Rev., 128, 2165-2189.

—, R. B. Wilhelmson, L. J. Wicker, and C. L. Ziegler, 2004: Numerical simulation of the interaction between the dryline and horizontal convective rolls. Mon. Wea. Rev., 132, 1792 1812.

Purdom, J. F. W., 1982: Subjective interpretations of geostationary satellite data for nowcasting. Nowcasting, K. Browning, Ed., Academic Press, 149-166.

Rotunno, R., J. B. Klemp, and M. L. Weisman, 1988: A theory for strong, long-lived squall lines. J. Atmos. Sci., 45, 463-485.

Schlesinger, R. E., 1984: Effects of the pressure perturbation field in numerical models of unidirectionally sheared thunderstorm convection: Two versus three dimensions. J. Atmos. Sci., 41, 1571-1587.

Stull, R. B., 1985: A fair-weather cumulus cloud classification scheme for mixed-layer studies. J. Climate Appl. Meteor., 24, $49-56$.

Weckwerth, T. M., and D. B. Parsons, 2006: A review of convection initiation and motivation for IHOP_2002. Mon. Wea. Rev., 134, 5-22.

Weisman, M. L., and R. Rotunno, 2004: "A theory for strong long-lived squall lines" revisited. J. Atmos. Sci., 61, 361-382.

Wicker, L. J., and R. B. Wilhelmson, 1995: Simulation and analysis of tornado development and decay within a threedimensional supercell thunderstorm. J. Atmos. Sci., 52, 26752703.

_ and W. C. Skamarock, 1998: A time-splitting scheme for the elastic equations incorporating second-order Runge-Kutta time differencing. Mon. Wea. Rev., 126, 1992-1999.

_ M. P. Kay, and M. P. Foster, 1997: STORMTIPE-95: Results from a convective storm forecast experiment. Wea. Forecasting, 12, 388-398.

Wilson, J. W., and W. E. Schreiber, 1986: Initiation of convective storms at radar-observed boundary-layer convergence lines. Mon. Wea. Rev., 114, 2516-2536.

- and R. D. Roberts, 2006: Summary of convective storm initiation and evolution during IHOP: Observational and modeling perspective. Mon. Wea. Rev., 134, 23-47.

Xu, Q., 1992: Density currents in shear flows-A two-fluid model. J. Atmos. Sci., 49, 511-524.

_ , M. Xue, and K. K. Droegemeier, 1996: Numerical simulations of density currents in sheared environments within a vertically confined channel. J. Atmos. Sci., 53, 770-786.

Xue, M., 2002: Density currents in shear flows: Effects of rigid lid and cold-pool internal circulation, and application to squall line dynamics. Quart. J. Roy. Meteor. Soc., 128, 47-74.

—, Q. Xu, and K. K. Droegemeier, 1997: A theoretical and numerical study of density currents in nonconstant shear flows. J. Atmos. Sci., 54, 1998-2019.

Ziegler, C. L., and E. N. Rasmussen, 1998: The initiation of moist convection at the dryline: Forecasting issues from a case study perspective. Wea. Forecasting, 13, 1106-1131.

_ T. J. Lee, and R. A. Pielke Sr., 1997: Convective initiation at the dryline: A modeling study. Mon. Wea. Rev., 125, 10011026. 